\title{
A Review of Exhaled Volatile Organic Compounds as Biomarkers for Thoracic Malignancies
}

\author{
Gerardo Velez ${ }^{*}$, Harvey Pass \\ Department of Cardiothoracic Surgery, NYU Langone Health, New York, USA \\ Email address: \\ gerardo.velez@nyumc.org (G. Velez), harvey.pass@nyumc.org (H. Pass) \\ ${ }^{*}$ Corresponding author
}

To cite this article:

Gerardo Velez, Harvey Pass. A Review of Exhaled Volatile Organic Compounds as Biomarkers for Thoracic Malignancies. American Journal of Biomedical and Life Sciences. Vol. 8, No. 6, 2020, pp. 231-247. doi: 10.11648/j.ajbls.20200806.17

Received: December 2, 2020; Accepted: December 10, 2020; Published: December 31, 2020

\begin{abstract}
Lung cancer, malignant pleural mesothelioma, and esophageal cancer are the most common thoracic malignancies and are responsible for substantial cancer-related morbidity and mortality worldwide. Early cancer identification prompts earlier intervention and can therefore improve patient survival. Traditional diagnostics are costly and invasive, however, creating an urgent need for alternative methods. Over the past 30 years, breath analysis has emerged as a rapid, minimally invasive, and cost-effective approach. Metabolites in exhaled breath, known as volatile organic compounds (VOCs), reflect internal biomolecular processes and their composition has been shown to vary in association with numerous pathological states. This review provides an overview on the use of VOCs in exhaled breath for the early screening and diagnosis of thoracic malignancies. Study design, methodology, and significant results from over sixty studies published since 1990 are specified and summarized. A total of 439 significant VOCs are reported in the literature, mainly consisting of aromatic compounds, aldehydes, alkanes, lipids, ketones, and sulfur-containing compounds. Diagnostic sensitivities and specificities range from $51-100 \%$ and $68.8-100 \%$, respectively. Cancer-specific VOC profiles and associations of clinical interest (e.g., comorbidities, histology, and staging) are emphasized and discussed. While there is considerable evidence to support the diagnostic utility of VOCs, the lack of standardization and external validation in large independent cohorts remain key barriers to clinical translation. However, efforts to address these limitations are currently underway.
\end{abstract}

Keywords: Lung Cancer, Esophageal Cancer, Mesothelioma, Volatile Organic Compounds, VOCs, Breath Analysis, Biomarker

\section{Introduction}

Thoracic malignancies are aggressive neoplasms of uncontrolled cell growth that originate within the chest cavity. Lung cancer, malignant pleural mesothelioma, and esophageal cancer are the most common thoracic malignancies. Early screening and detection can reduce mortality in all three cancers [1-3]. Current methods of investigation rely on a combination of blood, imaging, and tissue sampling techniques that are invasive, inaccurate, and/or costly [3-8]. Over the past few decades, volatile organic compounds (VOCs) in exhaled breath have emerged as a potentially quick, safe, noninvasive biomarker for detecting lung cancer, esophageal cancer, and malignant pleural mesothelioma. Prior reviews have considered these three malignancies separately; overall VOC patterns between lung cancer, esophageal cancer, and malignant pleural mesothelioma have not been explored.

The aim of this review is to consolidate the current knowledge on exhaled VOCs in the early screening and diagnosis of thoracic malignancies. Literature published between January 1990 and May 2020 is included. In the following sections, we will summarize breath composition and findings of available studies on lung cancer, esophageal cancer, and mesothelioma. Clinically relevant VOCs will be emphasized, along with sensitivities and specificities. Focused reviews on study design and methodology of the three respective cancers can be found elsewhere. We will conclude with a discussion on general VOC patterns between all three thoracic cancers along with potential biological mechanisms. 


\section{Background}

\subsection{Thoracic Malignancies}

\subsubsection{Lung Cancer}

Lung cancer (LC) is a tumor of lung tissue most commonly caused by smoking cigarettes [9]. Other risk factors include second-hand smoke, air pollution, and asbestos. Lung cancer is the leading cause of cancer mortality in the United States and worldwide, accounting for 2.1 million new cases and 1.8 million deaths in 2018 [10]. Over 50\% of patients with lung cancer die within one year following diagnosis and have a five-year survival rate of less than $20 \%$ [9].

\subsubsection{Malignant Pleural Mesothelioma}

Malignant pleural mesothelioma (MPM) is an aggressive neoplasm originating from the mesothelial cells lining the pleura and is causally associated with previous asbestos exposure [11]. Malignant pleural mesothelioma has a median latency time of 40-50 years between first exposure to asbestos and its diagnosis. Once diagnosed, the majority of patients die within 2 years. Malignant pleural mesothelioma is rare in the United States, however, accounting for about 3,000 new cases diagnosed each year. Globally, malignant pleural mesothelioma accounts for an estimated 38,400 cancer related deaths per year, with mortality rates increasing [12].

\subsubsection{Esophageal Cancer}

Esophageal (or esophagogastric) cancer (EC) is a malignant proliferation of mucosal cells lining the esophagus, gastroesophageal junction, and parts of the stomach. Smoking, drinking alcohol, and gastroesophageal reflux disease (GERD) are among the most common risk factors associated with developing esophageal cancer [13]. Esophageal cancer is the 6th leading cause of cancer related mortality death worldwide, affecting approximately 1.5 million a year and $15 \%$ of cancer related deaths [14]. Esophageal cancer makes up about 1\% of all cancers diagnosed in the United States, but it is much more common in some other parts of the world, such as Iran, northern China, India, and southern Africa [15].

\subsection{Volatile Organic Compounds}

Volatile organic compounds (VOCs) are organic compounds with relatively high vapor pressure or volatility, that can be detected in the headspace of cancer cells, blood samples, and/or in various bodily excrements, e.g., sweat, urine, feces, and breath [16]. VOCs reflect cellular processes and metabolic activity at the tissue level, such as inflammation and oxidative stress, and can therefore function as a surrogate marker of various states of health and disease (Figure 1) [17]. VOCs enter the bloodstream and travel to the lungs, where they diffuse across the pulmonary alveolar membrane and are exhaled through the breath.

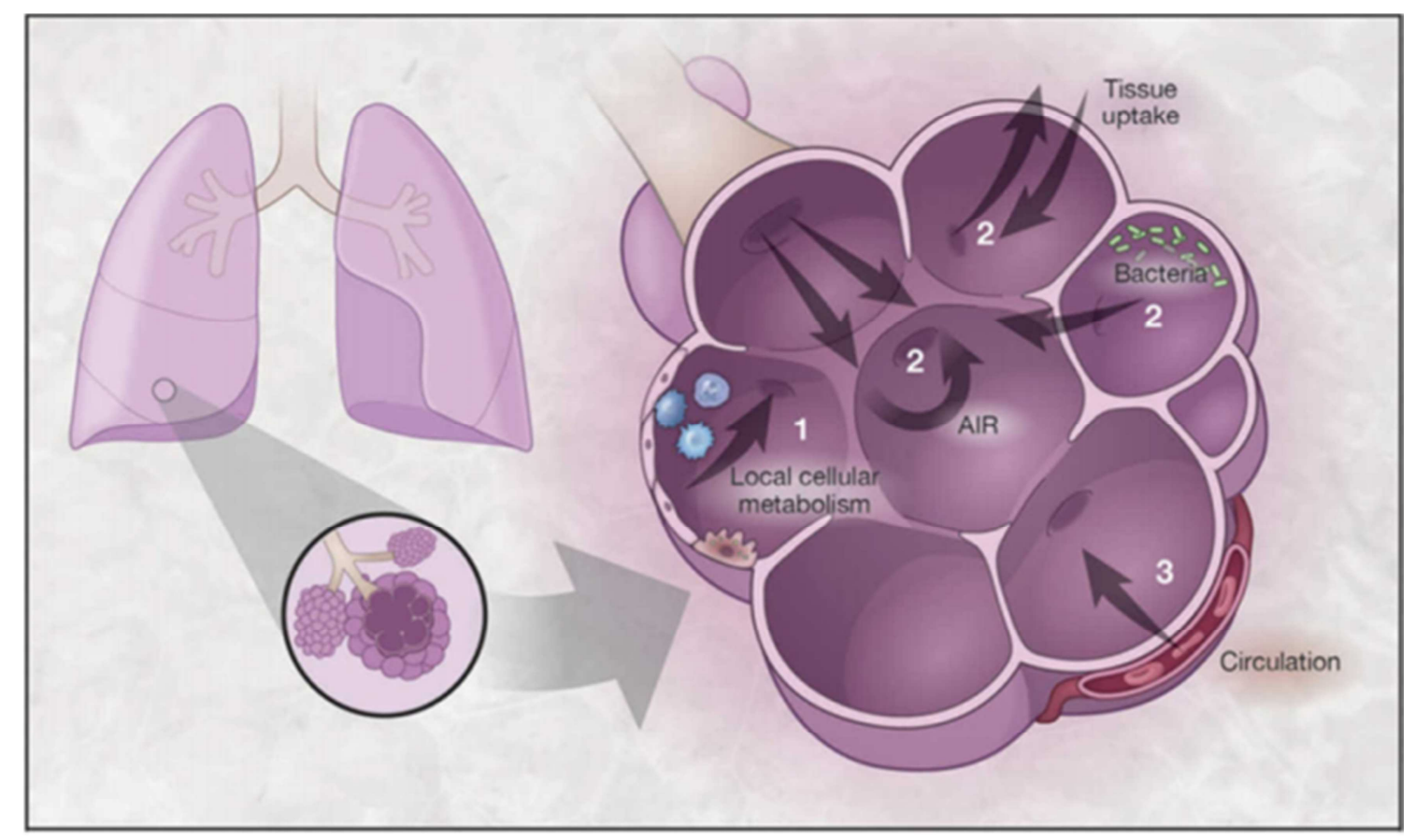

Figure 1. Diagram of volatile organic compounds (VOCS) in the lung. VOCs in exhaled breath originate from three primary sources: (1) local cellular metabolism from airways and alveoli, (2) inhaled from the surrounding environment or produced by resident bacteria, or (3) elsewhere in the body, subsequently carried to the lungs via bloodstream. Reprinted from "Breathomics in Lung Disease "by van der Schee MP, et al., 2015, Chest, 147 (1): 224-231, Copyright 2015, with permission from Elsevier.

The most abundant VOCs in human breath are acetone, methanol, ethanol, propanol and isoprene [18]. On average, a single breath sample contains around 200 different VOCs [19]. Changes in VOC composition and/or concentration can therefore signal underlying pathology and be used to guide clinical practice. To date, breath VOCs have shown clinical value in the diagnosis and management of numerous diseased states, including Inflammatory Bowel Disease (IBD) [20], 
infectious diseases [21-23], Asthma [24-26], Cystic Fibrosis [25], Chronic Obstructive Pulmonary Disease (COPD) [25, 26], Alzheimer's [27], and various cancers [28-30].

The use of VOCs for lung cancer screening and detection has been investigated in dozens of studies over the past 30 years. More recently, VOC biomarker studies on esophageal cancer and malignant pleural mesothelioma have begun to surface as well. However, despite the sizeable body of literature, a consensus list of validated VOCs does not exist.

\subsection{Methodologic Principles}

VOC studies primarily concern themselves with two distinct but related outcomes. Characterization studies seek to discover VOCs significantly associated with cancer, while diagnostic studies evaluate the ability of VOC profiles to determine the presence of cancer. While the latter approach is of greater clinical interest, the approaches are complimentary and a majority of studies employ both.

Many techniques and technologies have been developed over the years to enhance VOC extraction and analysis [31].

\subsubsection{Breath Sampling}

Exhaled breath is typically either collected and analyzed immediately or stored in containers for transport to a VOC detector. Given the presence of trace VOC concentrations, breath samples often undergo preconcentration with solid-phase microextraction (SPME) to improve VOC detection. The most common VOC containers are sampling bags (e.g., Tedlar, Mylar). Sampling bags are cheap, chemically inert, and readily interface with numerous other lab and clinical equipment for preconcentration and/or detection. However, sampling bags are susceptible to leakage, UV degradation, and water condensation.

Once exhaled breath samples are collected and preconcentrated, they undergo VOC detection and profiling.

\subsubsection{VOC Detection}

VOC detection is primarily based on mass spectrometry and electronic nose (e-nose) technologies (Figure 2).

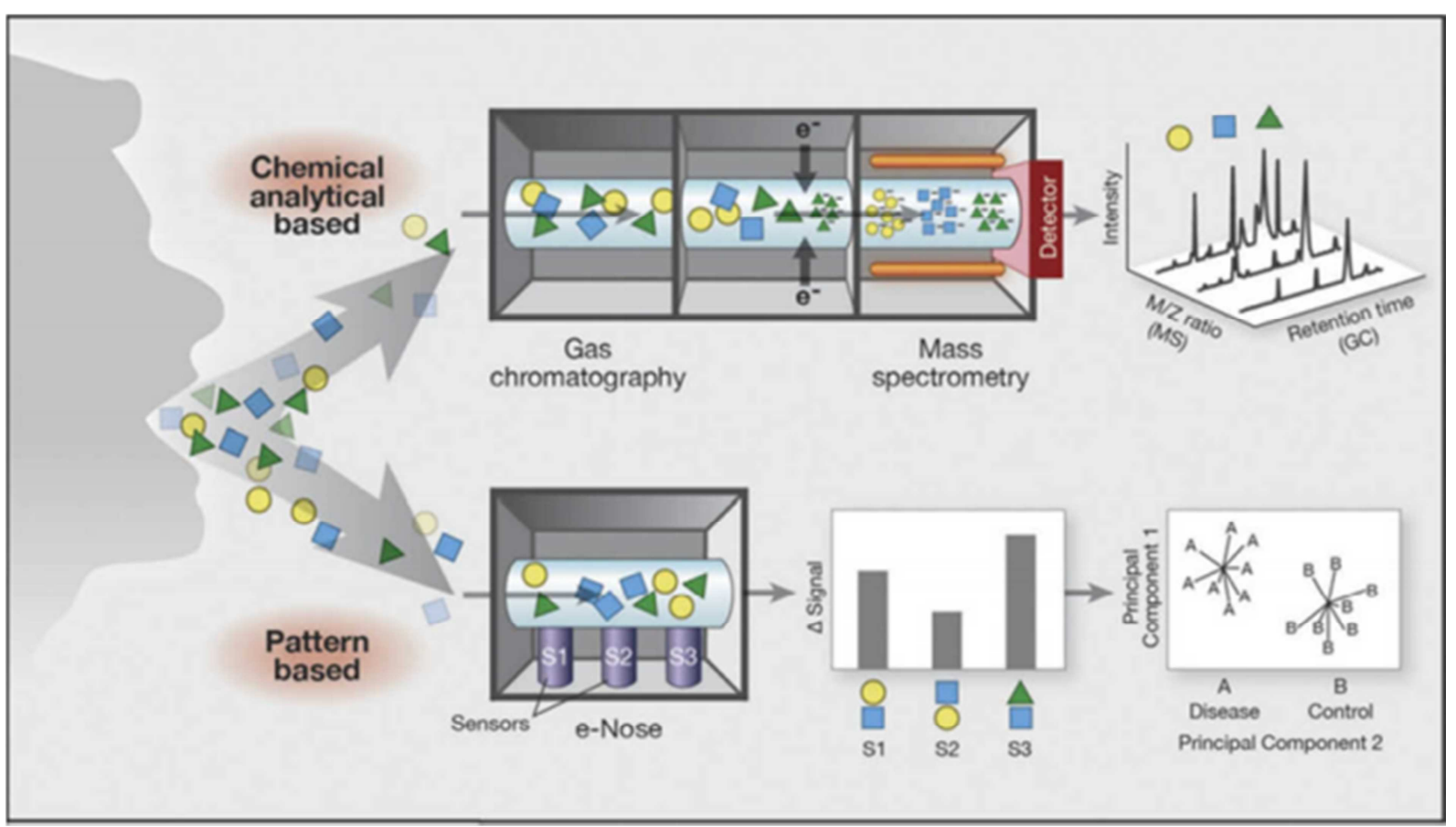

Figure 2. Detection and analysis of exhaled VOCs. Left: Exhaled VOCs are a complex mixture of different compounds (depicted by triangle, circle, and square symbols). The chemical analysis approach is represented by GC-MS, which separates VOCs and allows identification based on mass/charge ratios. The pattern approach is represented by e-nose. Cross-reactive sensors bind to VOCs and generate a characteristic composite signal, which then undergoes classification by pattern-recognition algorithms. Reprinted from "Breathomics in Lung Disease "by van der Schee MP, et al., 2015, Chest, 147 (1): 224-231, Copyright 2015, with permission from Elsevier.

Gas chromatography-mass spectrometry (GC-MS) is the current gold standard for VOC analysis. Compounds in exhaled breath are separated by gas chromatography and ionized in the mass spectrometer. VOCs can then be identified based on their mass/charge ratios. GC-MS allows for chemical identification and quantification of VOCs.

However, GC-MS technology is slow, expensive, labor intensive, and requires skilled operation. E-nose is rapidly emerging as a more practical alternative for VOC detection and essentially rely on pattern recognition. Sensors react to
VOCs in exhaled breath and produce electrical signals, which combine to generate a composite pattern of surrounding air components, or "breathprint," that can be further analyzed.

Unlike GC-MS, selective characterization and identification of VOCs is not possible. However, sensor arrays are cheaper, portable, and provide real-time results, making them more suitable for point-of-care use than GC-MS.

\subsubsection{VOC Analysis}

VOC analysis typically evaluates the breath composition of cancer and benign subjects to characterize malignancy using 
two main approaches: (1) VOC identification, and (2) VOC patterning. VOC identification uses GC-MS to generate a differential panel of known and discrete VOCs, while VOC patterning uses e-nose to generate a discriminant breathprint from unknown and aggregate VOCs. In either case, rigorous statistical analyses can be used to develop a VOC biosignature of malignant disease and educate diagnostic models.

\section{Thoracic Malignancies \& VOCs}

Our review included 66 publications since 1990 using exhaled breath VOCs to determine thoracic cancer.

In general, studies sought to identify VOCs uniquely associated with thoracic cancer $(n=39)$, and/or test the ability of VOCs to determine malignancy $(n=61)$. Within included studies, lung $(n=53$, Table 1$)$, mesothelioma $(n=7$, Table 2$)$, and esophagogastric $(n=6$, Table 3$)$ cancers were the primary cancers of interest. One study investigated VOCs as biomarkers for both lung cancer and mesothelioma. Studies typically compared patients with thoracic cancer against a healthy control population and/or patients with benign conditions. Cancer cohorts were often of mixed histological subtype and staging. MS-based techniques were the most common analytical platforms $(n=42$ studies) followed by electronic sensor-based $(n=28)$.

A total of 439 significant VOCs were reported in association with diagnosis for thoracic cancers (Tables 4-6). For the most part, these VOCs were aromatic compounds, aldehydes, alkanes, lipids, ketones, and sulfur-containing compounds (Figure 3). The most common VOCs associated with thoracic cancer were benzene $(n=10$ studies $)$, ethylbenzene $(n=9)$, hexanal $(n=9)$, isoprene $(n=9)$, propanol $(n=8)$, styrene $(n=8)$, propylbenzene $(n=7)$, and xylene $(n=7)$.

Sensitivities and specificities ranged from 51-100\% and 11 $-100 \%$, respectively. Diagnostic VOC panels, ranging from 1 to 500 VOCs, were described and identified in 37 studies.

Table 1. Summary of VOC studies on lung cancer

\begin{tabular}{|c|c|c|c|}
\hline Author & Cohorts & Histology NSCLC (Adeno, SCC), SCLC, other & Staging (I, II, III, IV) \\
\hline Bajtarevic et al. [32] & 65 LC vs. $31 \mathrm{H} 220$ LC vs. $441 \mathrm{H}$ & $47(25,17), 15,3$ & NR \\
\hline Buszewski et al. [33] & 29 LC vs. $44 \mathrm{H}$ & 110,18 & NR \\
\hline Broza et al. [34] & 12 LC vs 5 PN & NR & $9,3,0,0$ \\
\hline Cai et al. [35] & $57 \mathrm{LC}$ vs. $72 \mathrm{H}$ & 53()$, 4$ & NR \\
\hline Chang et al. [36] & $37 \mathrm{LC}$ vs. $48 \mathrm{H}$ & $36(27,9), 0,1$ & $25,6,5,1$ \\
\hline Chen et al. [37] & 29 LC vs. $20 \mathrm{~B}$ & $25(19,6), 4$ & NR \\
\hline Chen et al. [38] & $5 \mathrm{LC}$ vs. $5 \mathrm{H}$ [50 training] & NR & NR \\
\hline D'Amico et al. [40] & $28 \mathrm{LC}$ vs. $36 \mathrm{H}$ vs. $28 \mathrm{CM}$ & $24(18,6), 4$ & NR \\
\hline Di Natale et al. [41] & 35 LC vs. $27 \mathrm{~B}$ & NR & NR \\
\hline Dragonieri et al. [42] & $10 \mathrm{LC}$ vs. $10 \mathrm{CM}$ & $10(4,4)$ & $5,1,4,0$ \\
\hline Fu et al. [43] & 97 LC vs. $32 \mathrm{PN}$ & $88(33,32), 9$ & $34,16,24,115 \mathrm{LD}, 4 \mathrm{ED}$ \\
\hline Fuchs et al. [44] & 12 LC vs. $24 \mathrm{H}$ & 8()$, 4$ & $12 \mathrm{~L}$ \\
\hline Gaspar et al. [45] & $18 \mathrm{LC}$ vs. $10 \mathrm{H}$ & 17()$, 1$ & $0,0,0,18$ \\
\hline Gasparri et al. [46] & $21 \mathrm{LC}$ vs. $20 \mathrm{~B}$ [105 training] & $56(42,14), 0,14$ & $40,18,6$ (III+IV) \\
\hline Handa et al. [47] & $50 \mathrm{LC}$ vs. $39 \mathrm{H}$ & $42(32,10), 8$ & $13,6,8,23$ \\
\hline Hubers et al. [49] & 18 LC vs. 8 B [51 training] & $28(14,10), 6,4$ & $5,5,14,14$ \\
\hline Kischkel et al. [50] & $31 \mathrm{LC}$ vs. $62 \mathrm{H}$ & 19()$, 12$ & NR \\
\hline Kononov et al. [51] & $65 \mathrm{LC}$ vs. $53 \mathrm{H}$ & 59()$, 6$ & $30 \mathrm{E}, 35 \mathrm{~L}$ \\
\hline Kort et al. [52] & 144 LC vs. 146 B & $144(93,42), 18$ & $22 \mathrm{E}, 122 \mathrm{~L}$ \\
\hline Ligor et al. [53] & $65 \mathrm{LC}$ vs. $31 \mathrm{H}$ & 47()$, 15,3$ & NR \\
\hline Lu et al. [54] & 98 LC vs. 116 B & $86(48,38), 12$ & $2,6,44,46$ \\
\hline Machado et al. [55] & 14 LC vs. 62 B [59 training] & 21()$, 7$ & $4,1,8,94 \mathrm{LD}, 2 \mathrm{ED}$ \\
\hline Mazzone et al. [56] & $83 \mathrm{LC}$ vs. $137 \mathrm{~B}$ & $83(50,23), 9$ & $32,9,19,23$ \\
\hline Mazzone et al. [57] & 49 LC vs. 94 B & $49(27,13)$ & $14,4,15,16$ \\
\hline Molina et al. [58] & 68 vs. $126 \mathrm{~B}$ & $46(13,20), 9,13$ & $14 \mathrm{E}, 54 \mathrm{~L}$ \\
\hline Oguma et al. [59] & $116 \mathrm{LC}$ vs. $37 \mathrm{H}$ & $91(55,18,18), 25$ & $9,2,47,58$ \\
\hline Peled et al. [60] & 53 LC vs 19 PN & $45(30,13), 5$ & $23,4,10,103 \mathrm{LD}, 3 \mathrm{ED}$ \\
\hline Phillips et al. [61] & 193 LC vs. $211 \mathrm{H}$ & 189()$, 4$ & $96,24,31,17$ \\
\hline Phillips et al. [62] & 65 LC vs. 96 PN [301 training] & NR & NR \\
\hline Phillips et al. [63] & 73 LC vs. 142 B [301 training] & $161(108,29) 1,6$ & NR \\
\hline Phillips et al. [66] & 68 LC vs. 48 B & $50(23,24), 10,2$ & $9,3,18,27,3 \mathrm{X}$ \\
\hline Poli et al. [67] & $36 \mathrm{LC}$ vs. $110 \mathrm{~B}$ & 36() & $36 \mathrm{E}$ \\
\hline Rocco et al. [68] & 23 LC vs. $77 \mathrm{~B}$ & $23(18,5)$ & NR \\
\hline Rudnicka et al. [69] & $108 \mathrm{LC}$ vs. $121 \mathrm{H}$ & 74()$, 15,19$ Other & $5,12,22,3515 \mathrm{ED}$ \\
\hline Rudnicka et al. [70] & $23 \mathrm{LC}$ vs. $30 \mathrm{H}$ & $18(3,15), 2,3$ & NR \\
\hline Sakumura et al. [71] & $107 \mathrm{LC}$ vs. $29 \mathrm{H}$ & NR & $55,15,28,9$ \\
\hline Schallschmidt, [72] & $37 \mathrm{LC}$ vs. $23 \mathrm{H}$ & $30(16,11), 7$ & $9,9,10,9$ \\
\hline Shehada et al. [73] & 149 LC vs. 56 CM & NR & $34 \mathrm{E}, 110 \mathrm{~L}$ \\
\hline
\end{tabular}




\begin{tabular}{llll}
\hline Author & Cohorts & Histology NSCLC (Adeno, SCC), SCLC, other & Staging (I, II, III, IV) \\
\hline Shlomi et al. [74] & 16 early LC vs. 30 PN [73 late LC] & $82(73,9), 7$ & $9,7,20,53$ \\
Song et al. [75] & 43 LC vs. 41 H & $43(19,24)$ & $13,7,6,17$ \\
Tirzite et al. [76] & 252 LC vs. 223 B & $208(85,87), 35,9$ & $42,32,85,54$ \\
Tirzite et al. [77] & 45 LC vs. 39 B [251 training] & NR & LD, 34 ED \\
Ulanowska et al. [78] & 137 LC vs. 143 H & $98(35,60), 33,6$ & NR \\
Van der Goor et al. [79] & 8 LC vs. 14 H [58 training] & 44()$, 7,1$ & $4,2,18,28$ \\
Wang et al. [80] & 233 LC vs. 140 H vs. 111 CM & NR & NR \\
Wang et al. [81] & 85 LC vs. 158 B & $79(38,29), 4,2$ & $16,11,25,29$ \\
Westhoff et al. [82] & 32 LC vs. 54 H & $24(0,7,6$ & $0,3,9,17$ \\
Yu et al. [83] & 15 LC vs. 15 H & $13(10,3), 2$ & $0,4,9,2$ \\
Zou et al. [84] & 58 LC vs. 20 B [171 training] & $111(67,44), 12,14$ & $27,16,36,464 \mathrm{LD}, 8 \mathrm{ED}$ \\
\hline
\end{tabular}

Table 1. Continued.

\begin{tabular}{|c|c|c|c|c|}
\hline Author & Sampling Method & Detectio Method & Key Biomarkers & Sensitivity/Specificity \\
\hline Bajtarevic et al. [32] & Bag, SPME & GC-MS, PTR-MS & $21 \mathrm{VOCs}$ & $80.0 / 100.0 \%$ \\
\hline Buszewski et al. [33] & Bag, SPME & Canines, GC-TOF-MS & Profile & $82.20 / 82.40 \%$ \\
\hline Broza et al. [34] & Bag, SPME & e-nose, GC-MS & Profile & $100.0 / 80.0 \%$ \\
\hline Cai et al. [35] & Bag & e-nose & 3 VOCs & $76.0 / 94.0 \%$ \\
\hline Chang et al. [36] & Bag, TD & e-nose & Profile & $79.0 / 72.0 \%$ \\
\hline Chen et al. [37] & Bag, SPME & GC-FID/MS & $11 \mathrm{VOCs}$ & $86.2 / 70.0 \%$ \\
\hline Corradi et al. [39] & BioVOC tube, SPME & GC-MS & 6 VOCs $(+\mathrm{EBC})$ & $72.5 / 75.8 \%$ \\
\hline D'Amico et al. [40] & Bag, SPME & e-nose, GS-MS & Profile & $89.3 / 75.0 \%$ \\
\hline Di Natale et al. [41] & Bag & e-nose & Profile & $100.0 / 94.0 \%$ \\
\hline Dragonieri et al. [42] & VOC filter, Bag & e-nose & Profile & NR ( $85 \%$ accuracy) \\
\hline Fu et al. [43] & Bag, Silicon Microreactor & FT-ICR MS & 2-4 VOCs & $89.8 / 81.3 \%$ \\
\hline Fuchs et al. [44] & GTS, HSV, SPME & GC-MS & $4 \mathrm{VOCs}$ & $75.0 / 95.8 \%$ \\
\hline Gasparri et al. [46] & Mouthpiece, Bag & e-nose & Profile & $81.0 / 100.0 \%$ \\
\hline Handa et al. [47] & $\mathrm{CO} 2$-controlled inlet & IMS-MCC & $10 \mathrm{VOCs}$ & $76.0 / 100.0 \%$ \\
\hline Huang et al. [48] & ETT, Capnometer, Bag & e-nose & Profile & $83.3 / 86.2 \%$ \\
\hline Hubers et al. [49] & VOC Filter, Bag & e-nose & Profile & $94.0 / 13.0 \%$ \\
\hline Kischkel et al. [50] & GTS, HSV, SPME & GC-MS & $12 \mathrm{VOCs}$ & NR \\
\hline Kononov et al. [51] & Vapor dilution & e-nose & Profile & $95.0 / 100.0 \%$ \\
\hline Kort et al. [52] & VOC filter, TD & e-nose & Profile & $94.4 / 32.9 \%$ \\
\hline Ligor et al. [53] & GTS, Bag, SPME & GC-MS & $8 \mathrm{VOCs}$ & $51.0 / 100.0 \%$ \\
\hline Lu et al. [54] & Bag & e-nose & Profile & $94.2 / 92.8 \%$ \\
\hline Machado et al. [55] & MB, Bag, TD & e-nose, GC-MS & Profile & $71.4 / 91.9 \%$ \\
\hline Mazzone et al. [56] & Corrugated tubing & e-nose & Profile & $70.0 / 86.0 \%$ \\
\hline Mazzone et al. [57] & NR & e-nose & Profile & $73.3 / 72.4 \%$ \\
\hline Molina et al. [58] & ECOScreen2 & GC-TOF/MS & 5 VOCs & $86.8 / 67.5 \%$ \\
\hline Oguma et al. [59] & Double cold trap & GC-FID/MS & 2 VOCs (either) & $75.0 / 78.0 \%$ \\
\hline Phillips et al. [62] & BCA, ST, TD & GC-MS & $1 \mathrm{VOC}$ & $75.4 / 85.0 \%$ \\
\hline Phillips et al. [63] & $\mathrm{TD}, \mathrm{ST}$ & GC-MS & $500 \mathrm{VOCs}$ & $68.0 / 68.4 \%$ \\
\hline Phillips et al. [64] & Bag, SPME & GC-MS & $30 \mathrm{VOCs}$ & $84.5 / 81.0 \%$ \\
\hline Phillips et al. [65] & BCA, ST, TD & GC-MS & $9 \mathrm{VOCs}$ & $85.1 / 80.5 \%$ \\
\hline Phillips et al. [66] & BCA, ST, TD & GC-MS & $22 \mathrm{VOCs}$ & $71.7 / 66.7 \%$ \\
\hline Poli et al. [67] & Teflon bulb, SPME & GC-MS & 13 VOCs & $72.2 / 93.6 \%$ \\
\hline Rocco et al. [68] & Adsorbing Cartridge, TD & e-nose & Profile & $86.0 / 95.0 \%$ \\
\hline Rudnicka et al. [69] & Bag, SPME & GC-MS & 7 VOCs & $86.4 / 86.4 \%$ \\
\hline Rudnicka et al. [70] & Bag, SPME & GC-TOF/MS & $6 \mathrm{VOCs}$ & NR \\
\hline Sakumura et al. [71] & Bag, cold trap & GC-MS & $5 \mathrm{VOCs}$ & $95.0 / 89.0 \%$ \\
\hline Schallschmidt, [72] & Gas bulb, SPME & GC-MS & 19 VOCs & $92.0 / 96.0 \%$ \\
\hline Shehada et al. [73] & Bag & e-nose & Profile & $92.0 / 80.0 \%$ \\
\hline Shlomi et al. [74] & Bag, TD & e-nose & Profile & $75.0 / 93.3 \%$ \\
\hline Song et al. [75] & Bag, SPME & GC-MS & $1 \mathrm{VOC}$ & $93.0 / 92.7 \%$ \\
\hline Tirzite et al. [76] & Bag & e-nose & Profile & $96.2 / 90.6 \%$ \\
\hline
\end{tabular}




\begin{tabular}{lllll}
\hline Author & Sampling Method & Detectio Method & Key Biomarkers & Sensitivity/Specificity \\
\hline Tirzite et al. [77] & Bag & e-nose & Profile & $88.9 / 68.8 \%$ \\
Ulanowska et al. [78] & Bag, SPME & GC-MS & 14 VOCs & NR \\
Van der Goor et al. [79] & TD & e-nose & Profile & $88.0 / 86.0 \%$ \\
Wang et al. [80] & Bag, SPME, TD & GC-MS & 10 VOCs & $80.8 / 84.0 \%$ \\
Wang et al. [81] & Bag, SPME & GC-MS & 23 VOCs & $96.5 / 97.5 \%$ \\
Westhoff et al. [82] & Teflon bulb, Sampling loop & IMS-MCC & 23 VOCs & $100.0 / 100.0 \%$ \\
Yu et al. [83] & Bag, SPME & GC-FID & 5 VOCs & NR \\
Zou et al. [84] & BCA, Bag, SPME, TD & GC-MS & 5 VOCs & NR $(0.672-1.00$ AUC) \\
\hline
\end{tabular}

Adeno - Adenocarcinoma; AUC - area under the ROC curve; B - Benign group of both healthy and comorbid subjects; BCA - Breath collection apparatus; CM - Group with pulmonary comorbidities; ED - Extensive disease; ETT - Endotracheal tube; FID - Flame ionizing detector; FT - Fourier transform; GC - Gas chromatography; GTS - Gastight syringe; H - Healthy group with no comorbidities; HSV - Headspace Vial; ICR - Ion cyclotron resonance; IMS - Ion mobility spectrometry; LC - Lung cancer group; LD - Limited disease; MCC - Multi-capillary column; MS - Mass spectrometry; NR - Not reported; NSCLC Non-small cell lung cancer; PN - Group with benign pulmonary nodules; Profile - composite breathprint of VOCS; PTR - Proton transfer reaction; SCC Squamous cell carcinoma; SCLC - Small cell lung cancer; SPME - Solid phase microextraction; ST - Sorbent trap; TD - Thermal desorption; TOF - Time of flight; VOCs - Volatile organic compounds

Table 2. Summary of VOC studies on malignant pleural mesothelioma.

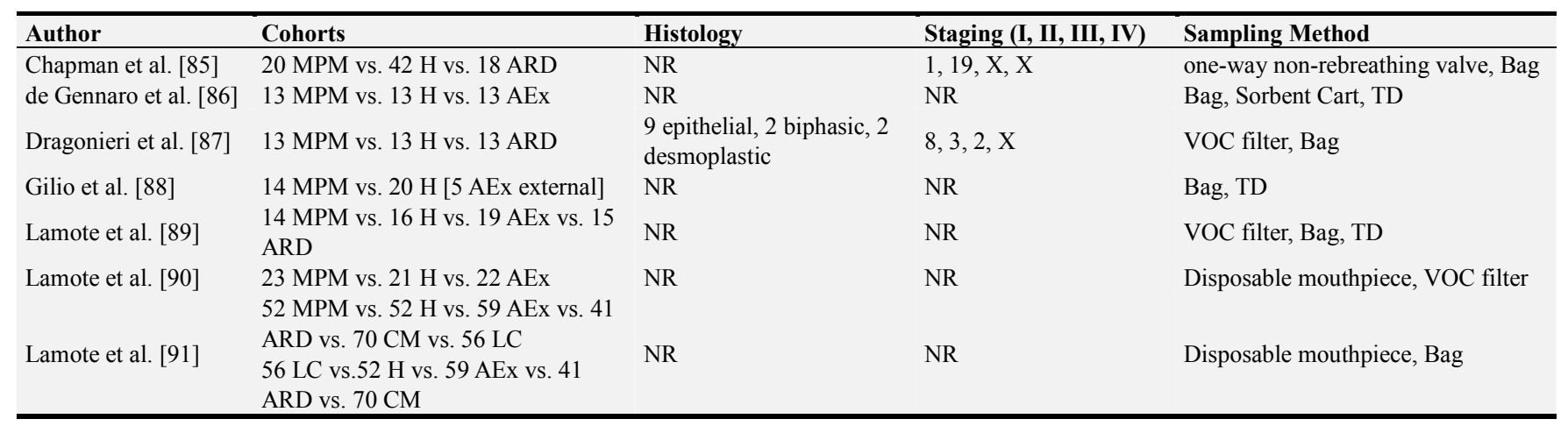

Table 2. Continued.

\begin{tabular}{|c|c|c|c|}
\hline Author & Detection Method & Key Biomarkers & Sensitivity/Specificity \\
\hline \multirow[t]{2}{*}{ Chapman et al. [85] } & e-nose & Profile (v. H) & $90 / 91 \%$ \\
\hline & & Profile (v. H v. ARD) & $90 / 88 \%$ \\
\hline de Gennaro et al. [86] & GC-MS & $11 \mathrm{VOCs}$ & $84.6 / 11 \%$ \\
\hline Dragonieri et al. [87] & e-nose & Profile (v. H) & $92 / 69 \%$ \\
\hline Gilio et al. [88] & GC-MS & $10 \mathrm{VOCs}$ & $100 / 50 \%$ \\
\hline \multirow[t]{9}{*}{ Lamote et al. [89] } & e-nose & Profile (v. ARD) & $92 / 86 \%$ \\
\hline & & Profile (v. H v. ARD) & NR ( $80 \%$ accuracy) \\
\hline & & Profile (v. AEx) & $80 / 64 \%$ \\
\hline & & Profile (v. ARD) & $75 / 64 \%$ \\
\hline & & Profile (v. Ex+ARD) & $82 / 55 \%$ \\
\hline & GC-MS & $8 \mathrm{VOCs}(\mathrm{v} . \mathrm{H})$ & $64 / 79 \%$ \\
\hline & & 17 VOCs (v. AEx) & $93 / 100 \%$ \\
\hline & & 7 VOCs (v. ARD) & $79 / 80 \%$ \\
\hline & & $19 \operatorname{VOCs}(\mathrm{v} . \mathrm{Ex}+\mathrm{ARD})$ & $100 / 91 \%$ \\
\hline \multirow[t]{3}{*}{ Lamote et al. [90] } & MCC-IMS & $2 \operatorname{VOCs}(\mathrm{v} . \mathrm{H})$ & $96 / 67 \%$ \\
\hline & & 6 VOCs (v. AEx) & $87 / 86 \%$ \\
\hline & & 4 VOCs (v. H+AEx) & $87 / 70 \%$ \\
\hline \multirow[t]{9}{*}{ Lamote et al. [91] } & MCC-IMS & $16 \operatorname{VOCs}(\mathrm{v} . \mathrm{H})$ & $89 / 42 \%$ \\
\hline & & 13 VOCs (v. AEx) & $87 / 90 \%$ \\
\hline & & 19 VOCs (v. ARD) & $89 / 73 \%$ \\
\hline & & 19 VOCs (v. Ex+ARD) & $94 / 80 \%$ \\
\hline & & 9 VOCs (v. CM) & $71.2 / 87 \%$ \\
\hline & & 32 VOCs (v. LC) & $73 / 71 \%$ \\
\hline & & $20 \operatorname{VOCs}(\mathrm{v} . \mathrm{H})$ & $77 / 65 \%$ \\
\hline & & 22 VOCs (v. AEx) & $89 / 90 \%$ \\
\hline & & 5 VOCs (v. CM) & $64.3 / 77 \%$ \\
\hline
\end{tabular}

AEx - Group with known asbestos exposure; ARD - Group with asbestos-related disease; AUC - area under the ROC curve; CM - Group with pulmonary comorbidities; GC - Gas chromatography; H - Healthy group with no comorbidities; IMS - Ion mobility spectrometry; LC - Lung cancer group; MCC Multi-capillary column; MPM - Malignant pleural mesothelioma; MS - Mass spectrometry; NR - Not reported; Profile - composite breathprint of VOCS; TD Thermal desorption; VOCs - Volatile organic compounds 
Table 3. Summary of VOC studies on esophageal cancer.

\begin{tabular}{llll}
\hline Author & Cohorts & Histology & Staging (I, II, III, IV) \\
\hline Chan et al. [92] & 66 BE vs. 56 B & NR & NR \\
Kumar et al. [93] & 18 EGC vs. 17 H vs. 18 CM & NR & NR \\
Kumar et al. [94] & 81 EGC vs. 129 B & 81 & 17, 20, 44, X \\
Markar et al. [95] & 163 EGC vs. 172 H & NR & NR \\
Peters et al. [96] & 129 BE vs. 132 H vs. 141 CM & NR & NR \\
Zou et al. [97] & 29 EC vs. 57 H & NR & Bag, GTS \\
\hline
\end{tabular}

Table 3. Continued.

\begin{tabular}{llll}
\hline Author & Detection Method & Key Biomarkers & Sensitivity/Specificity \\
\hline Chan et al. [92] & e-nose & Profile & $82 / 80 \%$ \\
Kumar et al. [93] & SIFT-MS & 4 VOCs & NR (0.91 AUC) \\
Kumar et al. [94] & SIFT-MS & 8 VOCs & $86.7 / 81.2 \%$ \\
Markar et al. [95] & SIFT-MS & 5 VOCs & $80 / 81 \%$ \\
Peters et al. [96] & e-nose & Profile (v. H), Profile (v. CM), Profile (v. B) & $57 / 67 \%, 64 / 74 \%, 91 / 74 \%$ \\
Zou et al. [97] & PTR-MS & 7 VOCs & $86.2 / 89.5 \%$ \\
\hline
\end{tabular}

AUC - area under the ROC curve; B - Benign group of both healthy and comorbid subjects; BE - Group with Barrett's esophagus; CM - Group with esophageal comorbidities; EGC - esophagogastric cancer; GC - Gas chromatography; GTS - Gastight syringe; H - Healthy group with no comorbidities; IMS - Ion mobility spectrometry; LC - Lung cancer group; MCC - Multi-capillary column; MPM - Malignant pleural mesothelioma; MS - Mass spectrometry; NR - Not reported; Profile - composite breathprint of VOCS; PTR - Proton transfer reaction; SIFT - Selected-ion flow-tube mass spectrometry; TD - Thermal desorption; VOCs Volatile organic compounds

Table 4. VOCs identified in lung cancer.

\begin{tabular}{|c|c|}
\hline Author & Significant VOCs \\
\hline $\begin{array}{l}\text { Bajtarevic et al. } \\
\text { [32] }\end{array}$ & $\begin{array}{l}21 \text { VOCs: 2-Butanone, Benzaldehyde, 2,3-Butanedione, 1-Propanol, 2-Butanone, 3-hydroxy-, 3-Butyn-2-ol, Butane, 2-methyl-, 2-Butene, } \\
\text { 2-methyl-, Acetophenone, 1-Cyclopentene, Methyl propyl sulfide, Urea, tetramethyl-, n-Pentanal, 1,3-Cyclopentadiene, 1-methyl-, 2-Butanol, } \\
\text { 2,3-dimethyl-, Isoquinoline, 1,2,3,4-tetrahydro-, Undecane, 3,7-dimethyl-, Benzene, cyclobutyl-, Butyl acetate, Ethylenimine, n-Undecane }\end{array}$ \\
\hline $\begin{array}{l}\text { Buszewski et al. } \\
\text { [33] }\end{array}$ & $\begin{array}{l}12 \text { VOCS: acetone, benzene, butanal, 2-butanone, ethyl acetate, ethyl benzene, furan, 2-pentanone, propanal, 1-propanol, 2-propanol, } 2 \\
\text { propenal }\end{array}$ \\
\hline Broza et al. [34] & 5 VOCs: 2-methyl-1-pentene, 2-hexanone, 3-heptanone, styrene, 2,2,4-Trimethyl-hexane \\
\hline Cai et al. [35] & 3 VOCs: hexane, 2,2,4,6,6-pentamethylheptane, 1,26-trimethylnaphthalene \\
\hline Chen et al. [37] & $\begin{array}{l}11 \text { VOCs: Styrene, Decane, Isoprene, Benzene, Undecane, 1-hexene, Hexanal, Propyl Benzene, 1,2,4-trimethyl benzene, Heptanal, Methyl } \\
\text { cyclopentane }\end{array}$ \\
\hline Chen et al. [38] & $\begin{array}{l}11 \text { VOCs: Styrene, Decane, Isoprene, Benzene, Undecane, 1-hexene, Hexanal, Propyl benzene, 1,2,4-trimethyl benzene, Heptanal, Methyl } \\
\text { Cyclopentane }\end{array}$ \\
\hline $\begin{array}{l}\text { Corradi et al. } \\
{[39]}\end{array}$ & 6 VOC: pentane, 2-methyl pentane, hexane, ethyl benzene, heptanal, trans-2-nonenal \\
\hline Fu et al. [43] & 4 VOCs: 2-butanone, 2-hydroxyacetaldehyde, 3-hydroxy-2-butanone, 4-hydroxyhexenal \\
\hline Fuchs et al. [44] & 4 VOCs: pentanal, hexanal, octanal, nonanal \\
\hline Gaspar et al. [45] & $\begin{array}{l}17 \text { VOCs: Nonadecane (n-C19H40), Eicosane (n-C20H42), Heneicosane (n-C21H44), Docosane (n-C22H46), 2-methyldodecane, } \\
\text { 2-methyltridecane, branched-pentadecane (C15H32), 2-methylpentadecane, 3-methyltridecane, Heptadecane (n-C17H36), } \\
\text { 2-methylundecane, 2-methyltetradecane, 3-methyltetradecane, Pentadecane (n-C15H32), 3-methylpentadecane, branched-hexadecane } \\
\text { (C16H34), 2-methylhexadecane }\end{array}$ \\
\hline Handa et al. [47] & $\begin{array}{l}11 \text { VOCs: n-Dodecane, 3-methy1-1=Butanol, 2-Hexanol, 2-Metylbutylacetat, Cyclohexanone, Iso-propylamin, n-Nonanal, Ethylbenzol, } \\
\text { Hexanal, Heptanal, 3-Methyl-1-butanol }\end{array}$ \\
\hline $\begin{array}{l}\text { Kischkel et al. } \\
\text { [50] }\end{array}$ & $\begin{array}{l}12 \text { VOCs: Acetonitrile, Benzene, 2,5-Dimethylfuran, Acetone, Butanal, Hexanal, Dimethyl sulfide, Dimethyl formamide, Toluene, Propanal, } \\
\text { Isopropanol, 1-Propanol }\end{array}$ \\
\hline Ligor et al. [53] & 8 VOCs: 1-propanol, 2-butanone, 3-butyn-2-ol, benzaldehyde, 2-methyl-pentane, 3-methyl-pentane, n-pentane, n-hexane \\
\hline $\begin{array}{l}\text { Machado et al. } \\
\text { [55] }\end{array}$ & 11 VOCs: Isobutane, Methanol, Ethanol, Acetone, Pentane, Isoprene, Isopropanol, Dimethylsulfide, Carbon disulfide, Benzene, Toluene \\
\hline Molina et al. [58] & $\begin{array}{l}10 \text { VOCs: p-cresol (4-methylphenol), eicosenamide, hexadecylindane, cumyl alcohol (2-Phenyl-2-propanol), } \\
\text { 2,4-bis-dimethylbenzyl-6-t-butylphenol, monostearin (Glycerol monostearate), spiro-2,4-heptane-1,5-dimethyl-6-methylene, } \\
\text { 13-heptadecyn-1-ol, methyl stearate, 2,6-bis-1,1-dimethylethyl-4-(1-methyl-1-phenylethyl)phenol }\end{array}$ \\
\hline Oguma et al. [59] & 2 VOCs: Cyclohexane, Xylene \\
\hline Peled et al. [60] & 1 VOC: 1 -octene \\
\hline $\begin{array}{l}\text { Phillips et al. } \\
\text { [61] }\end{array}$ & $\begin{array}{l}16 \text { VOCs: 1,5,9-Cyclododecatriene, 1,5,9-trimethyl-, Pentan-1,3-dioldiisobutyrate, 2,2,4-trimethyl, Benzoic acid, 4-ethoxy-, ethyl ester, } \\
\text { Propanoic acid, 2-methyl-, 1-(1,1-dimethylethyl)-2-methyl-1,3-propanediyl ester, 10,11-dihydro-5H-dibenz-(B,F)-azepine, } \\
\text { 2,5-Cyclohexadiene-1,4-dione, 2,6-bis(1,1-dimethylethyl)-, Benzene, 1,1-oxybis-, Furan, 2,5-dimethyl-, 1,1-Biphenyl, 2,2-diethyl-, } \\
\text { 3-Pentanone, 2,4-dimethyl-, trans-Caryophyllene, 1H-Indene, 2,3-dihydro-1,1,3-trimethyl-3-phenyl-, 1-Propanol, Decane, 4-methyl-, } \\
\text { 1,2-Benzenedicarboxylic acid, diethyl ester, 2,4-Hexadiene, 2,5-dimethyl- }\end{array}$ \\
\hline Phillips et al. & 30 VOCs: Isopropyl alcohol, 4-Penten-2-ol, Ethane, 1,1,2-trichloro-1,2,2-trifluouro-, Propane, 2-methoxy-2-methyl-, 1-Propene, \\
\hline
\end{tabular}




\begin{tabular}{|c|c|}
\hline Author & Significant VOCs \\
\hline [64] & $\begin{array}{l}\text { 1-(methylthio)-, (E)-, 2,3-Hexanedione, 5,5-Dimethyl-1,3-hexadiene, 3-Hexanone, 2-methyl-, 1H-Indene, 2,3-dihydro-4-methyl-,Camphor, } \\
\text { Bicyclo[2.2.1]heptan-2-one, 1,7,7-trimethyl-,(1S)-, 3-Cyclohexene-1-methanol, a,a4-trimethyl-, p-menth-1-en-8-ol, } \\
\text { 5-Isopropenyl-2-methyl-7-oxabicyclo[4.1.0]heptan-2-ol, a Isomethyl ionone, 2,2,7,7-Tetramethyltricyclo[6.2.1.0(1,6)]undec-4-en-3-one, } \\
\text { 2,2,4-Trimethyl-1,3-pentanediol diisobutyrate, Benzoic acid, 4-ethoxy-, ethyl ester, Bicyclo[3.2.2]nonane-1,5-dicarboxylic acid, 5-ethyl } \\
\text { ester, Pentanoic acid, 2,2,4-trimethyl-3-carboxyisopropyl, isobutyl ester, Propanoic acid, 2-methyl-, } \\
\text { 1-(1,1-dimethyl)-2-methyl-1,3-propanediyl ester, 1,2,4,5-Tetroxane, 3,3,6,6-tetraphenyl-, Benzophenone, 2,5-Cyclohexadien-1-one, } \\
\text { 2,6-bis(1,1-dimethylethyl)-4-ethylidene-, Furan, 2-[(2-ethoxy-3,4-dimethyl-2-cyclohexen-1-ylidene)methyl]-, Benzene, } \\
\text { 1,1-(1,2-cyclobutanediyl)bis-, cis-, Benzene, 1,1-[1-(ethylthio)propylidene]bis-, Anthracene, 1,2,3,4-tetrahydro-9-propyl-, } \\
\text { 9,10-Anthracenediol, 2-ethyl-, Benzene, 1,1-ethylidenebis[4-ethyl- }\end{array}$ \\
\hline $\begin{array}{l}\text { Phillips et al. } \\
\text { [65] }\end{array}$ & $\begin{array}{l}9 \text { VOCs: Butane, Tridecane 3-methyl, Tridecane 7-methyl, Octane 4-methyl, Hexane 3-methyl, Heptane, Hexane 2-methyl, Pentane, } \\
\text { 5-methyl Decane }\end{array}$ \\
\hline $\begin{array}{l}\text { Phillips et al. } \\
\text { [66] }\end{array}$ & $\begin{array}{l}22 \text { VOCs: Styrene, 2,2,4,6,6-pentaheptane, 2-methylheptane, decane, propylbenzene, undecane, methylcyclopentane, 1-methyl-2-pentyl } \\
\text { cyclopropane, trichlorofluoro methane, benzene, 1,2,4-trimethylbenzene, 2-methyl-(isoprene)1,3-butadiene, octane, 1-hexene, } \\
\text { 3-methylnonane, 1-heptene, 1,4-dimethylbenzene, 2,4-dimethylheptane, hexanal, cyclohexane, 1-methylethenylbenzene, heptanal }\end{array}$ \\
\hline Poli et al. [67] & $\begin{array}{l}13 \text { VOCs: Isoprene, 2-methylpentane, pentane, ethylbenzene, xylenes total, Trimethylbenzene, Toluene, Benzene, Heptane, Decane, } \\
\text { Styrene, Octane, Pentamethylheptane }\end{array}$ \\
\hline $\begin{array}{l}\text { Rudnicka et al. } \\
{[69]}\end{array}$ & 7 VOCs: Acetone, isoprene, methyl acetate, methyl vinyl ketone, cyclohexane, 2-methylheptane, cyclohexanone \\
\hline $\begin{array}{l}\text { Rudnicka et al. } \\
{[70]}\end{array}$ & 6 VOCs: isopropyl alcohol, styrene, carbon disulfide, ethylbenzene, 2-propenal, propane. \\
\hline $\begin{array}{l}\text { Sakumura et al. } \\
\text { [71] }\end{array}$ & 5 VOCs: Hydrogen cyanide, Methanol, Acetonitrile, Isoprene, 1-propanol \\
\hline $\begin{array}{l}\text { Schallschmidt, et } \\
\text { al. [72] }\end{array}$ & $\begin{array}{l}19 \text { VOCs: n-pentane, n-hexane, n-heptane, n-octane, n-dodecane, 3-methylpentane, cyclohexane, benzene, ethylbenzene, n-propylbenzene, } \\
\text { propanal, n-butanal, n-hexanal, n-octanal, n-nonanal, n-decanal, 1-butanol, 2-butanone, 2-pentanone }\end{array}$ \\
\hline Song et al. [75] & 2 VOCs: 1-butanol, 3-hydroxy-2-butanone \\
\hline $\begin{array}{l}\text { Ulanowska et al. } \\
\text { [78] }\end{array}$ & $\begin{array}{l}14 \text { VOCs: Pentanal, Hexanal, Nonane, Ethanol, Acetone, Butane, Dimethyl sulfide, Isoprene, Propanal, 1-propanol, 2-pentanone, furan, } \\
\text { o-xylene, Ethylbenzene }\end{array}$ \\
\hline Wang et al. [80] & $\begin{array}{l}11 \text { VOCs: heneicosane, 3-ethyltoluene, 1,2,3-trimethylbenzene, N-propylbenzene, propylcyclohexane, indane, 1-methyl-3-propylbenzene, } \\
\text { o-xylene, 4-methyl-2-pentanone, 5-methylindan and methylcyclohexane }\end{array}$ \\
\hline Wang et al. [81] & $\begin{array}{l}23 \text { VOCs: hexadecanal, eicosane, 5-(2-methyl-)propylnonane, 7-methylhexadecane, 8-methylheptadecane, 2,6- di-tert-butyl-, } \\
\text { 4-methylphenol, 2,6,11-trimethyldodecane, 3,7-dimethylpentadecane, nonadecane, 8-hexylpentadecane, 4-methyltetradecane, } \\
\text { 2,6,10-trimethyltetradecane, 5-(1-methyl-)propylnonane, 2-methylnaphthalene, 2-methylhendecanal, nonadecanol, 2-pentadecanone, } \\
\text { tridecanone, 5-propyltridecane, 2,6-dimethylnaphthalene, tridecane, 3,8-dimethylhendecane, 5-butylnonane }\end{array}$ \\
\hline Yu et al. [83] & 5 VOCs: Benzene, Styrene, Propyl benzene, Decane, n-Undecane \\
\hline Zou et al. [84] & 5 VOCs: nonane,5-(2-methyl-)propyl-; phenol,2,6-di-tert-butyl-,4-methyl-; dodecane,2,6,11- trimethyl-; hexadecanal; pentadecane,8-hexyl- \\
\hline
\end{tabular}

Table 5. Significant VOCs identified in malignant pleural mesothelioma.

\begin{tabular}{ll}
\hline Author & Significant VOCs \\
\hline de Gennaro & 17 VOCs: Cyclopentane, cyclohexane, dodecane, xylene, toluene, decane, methylcyclohexane, dimethylnonane, benzaldehyde, limonene, \\
et al. [86] & $\alpha$-Pinene, $\beta$-Pinene, 1,2-pentadiene, methyl-octane, styrene, trimethylbenzene, acetophenone \\
$\begin{array}{l}\text { Gilio et al. } \\
\text { [88] }\end{array}$ & $\begin{array}{l}\text { 10 VOCs: acetophenone, } \alpha \text {-pinene, 1-hexonol-2-ethyl, p-benzoquinone, 2,2,4,6,6-pentamethyl-heptane, 1-propanol, benzonitrile, benzene, } \\
\text { ethylbezene, toluene }\end{array}$ \\
& 41 VOCs: Nonane, Propylbenzene, Benzonitrile, Isoprene, Limonene, 3-methylpentane, 1,3-dichlorobenzene, Ethanol, 2-ethyl-1-hexanol, \\
Lamote et & $\begin{array}{l}\text { Nonanal, 2-methyl-1-propanol, Methylcyclopentane, Cyclohexane, 1,2,4-trichlorobenzene, Naphtalene, Phenol, Chloroform, Linalool, } \\
\text { al. [89] }\end{array}$ \\
& $\begin{array}{l}\text { Furfural, Bromobenzene, Beta-pinene, Diethyl ether, Hexane, Isothiocyanatocyclohexane, 1,2-dichlorobenzene, n-Butylbenzene, } \\
\text { Methylbenzoate, 1,2,3-trichlorobenzene, Tert-butylbenzene, m/p-xylene, 2,2,4-trimethylpentane, Hexamethyldisiloxane, VOC IK 679, VOC }\end{array}$ \\
& IK 720, VOC IK 931, VOC IK 1100, VOC IK 1287, VOC IK 1233, VOC IK 1309, VOC IK 1349, VOC IK 1493 \\
\hline
\end{tabular}

Table 6. Significant VOCs identified in esophageal cancer.

\begin{tabular}{ll}
\hline Author & Significant VOCs \\
\hline Kumar et al. [93] & 5 VOCs: Hexanoic acid, Phenol, Methyl phenol, Ethyl Phenol, Methanol \\
Kumar et al. [94] & 13 VOCs: Nonanal, Decanal, Butyric Acid, Hexanoic acid, Phenol, Methyl Phenol, Heptanal, Ethyl Phenol, Butanal, Pentanal, Hexanal, \\
& Pentanoic acid, Octanal \\
Markar et al. [95] & 5 VOCs: Butyric Acid, Pentanoic Acid, Hexanoic Acid, Butanal, Decanal \\
Zou et al. [97] & 12 VOCs: Dimethyl sulfide, Thioethanol, Phenol, 1,3-cycloheptadiene, Dimethylsulfone, 2-methyl-1,3-diazine, \\
\hline
\end{tabular}




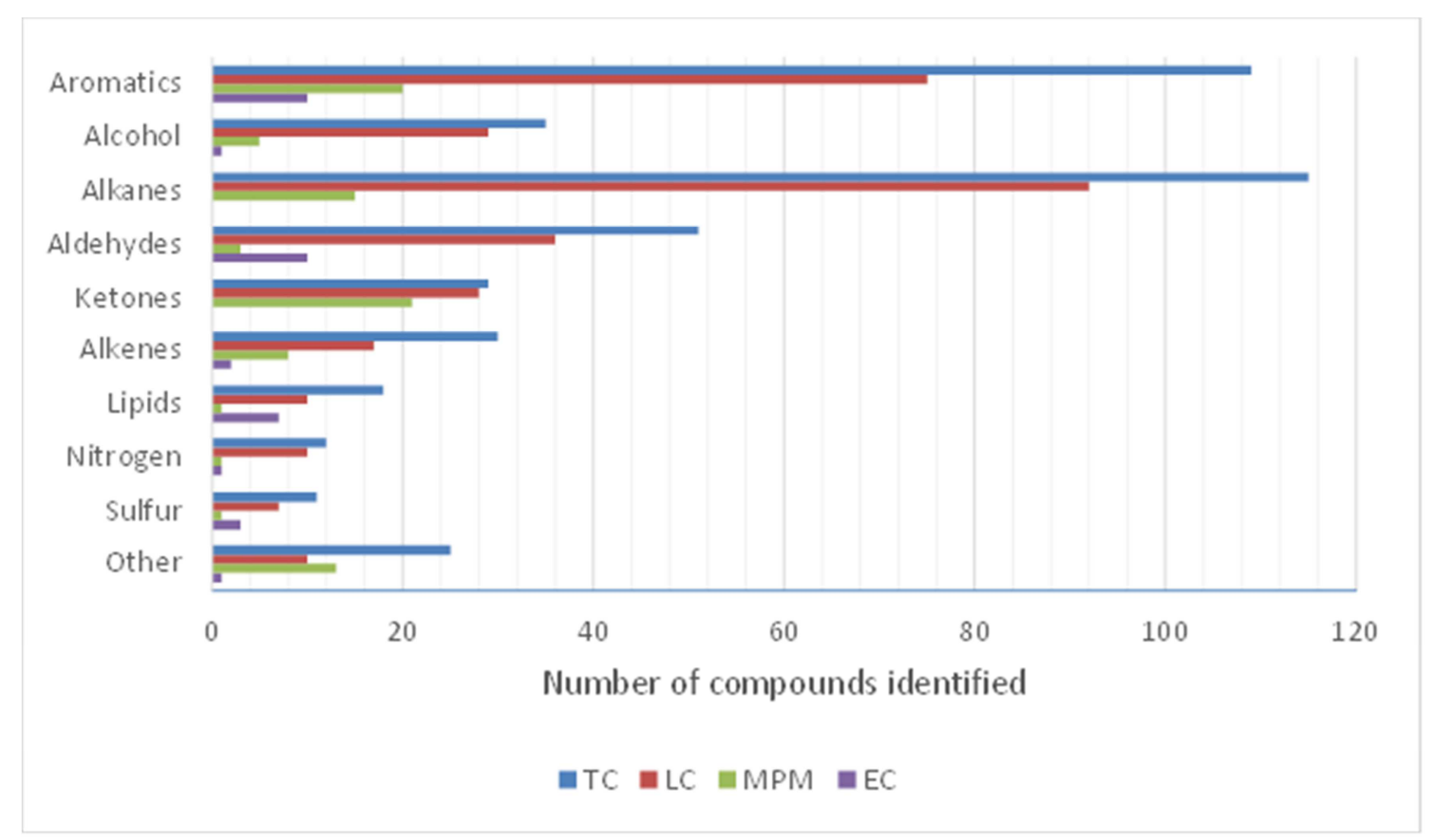

Figure 3. Summary of chemical classes of VOCs found in different thoracic cancers.

\subsection{Lung Cancer}

A total of 53 studies on LC and VOCs were reviewed.

VOCs uniquely associated with LC were reported in 32 studies. A total of 336 VOCs were reported as significant biomarkers for LC-mainly consisting of alkanes $(\sim 30 \%)$, aromatic compounds $(\sim 22 \%)$, and aldehydes $(\sim 11 \%)$ (Figure $3)$. The most common VOCs were Benzene, Styrene, Ethylbenzene, Propylbenzene, Propanol, 2-propanol, Pentane, Decane, Undecane, Hexanal, Heptanal, and Isoprene, which were used in at least 5 studies.

The ability of VOCs to determine malignancy was tested in 48 studies. Sensitivity and specificity ranged from $51-100 \%$ and $13.0-100 \%$, respectively. Diagnostic VOC models, ranging from 1 to 500 discrete VOCs, were described and evaluated in 26 studies. Five studies tested the ability to diagnose LC patients using a single VOC. Song et al. [75] showed lung cancer could be diagnosed using two univariate models: 1-butanol with $95.3 \%$ sensitivity and $85.4 \%$ specificity, and 3-hydroxy-2-butanone with 93\% sensitivity and $92.7 \%$ specificity. In a study with $233 \mathrm{LC}$ cases and 140 controls by Wang et al. [80], heneicosane was able to establish a diagnostic model with a sensitivity of $75.6 \%$, specificity of $78.9 \%$, and overall accuracy of $76.7 \%$. Oguma et al. [59] collected breath samples from $116 \mathrm{LC}$ cases and 37 controls and analyzed 14 VOCs with gas chromatography. Using cyclohexane alone, they achieved a sensitivity of $53 \%$ and a specificity of $78 \%$ for the diagnosis of lung cancer. Using xylene alone, they achieved a sensitivity of $49 \%$ and a specificity of $86 \%$. Using either cyclohexane or xylene, they achieved a sensitivity of $75 \%$ and a specificity of $78 \%$. Corradi et al. [39] examined breath samples from 71 LC cases and 67 controls, which included patients with comorbidities and pulmonary nodules. Trans-2-nonenol was able to establish a diagnosis of lung cancer with a sensitivity of $60.6 \%$ and a specificity of $62.7 \%$. In subgroup analyses of smoking exposure, sensitivity and specificity increased to $84.6 \%$ and $83.3 \%$ in patients with less than 10 pack-years exposure, and $75 \%$ and $73.3 \%$ in patients with less than 30 pack-years exposure. In a study by Molina et al. [58], p-cresol was able to discriminate between LC cases and $\mathrm{H}$ controls with $77.9 \%$ sensitivity and $74.2 \%$ specificity.

\subsubsection{Comorbid Cohorts}

Different disease states have been shown to alter the composition and concentration of VOCs in exhaled breath. Therefore, the presence of chronic conditions may complicate VOC detection and diagnosis of lung cancer. In addition to lung cancer, patients may also have hypertension, heart disease, and diabetes mellitus. Lung disease is particularly common and typically includes COPD, pulmonary nodules, and asthma. Pulmonary nodules may be benign or malignant, and early detection has been associated with improved survival [98].

i. Pulmonary Nodules

Four studies examined the ability to determine malignancy in the presence of PNs. Peled et al. [60] collected breath samples from 53 patients with malignant PNs and 19 patients with benign PNs with similar smoking histories and comorbidities. GC-MS analysis identified a significantly higher concentration of 1-octene in the breath of LC patients, and the nanoarray distinguished between malignant and benign PNs with $86 \%$ sensitivity and $96 \%$ specificity. Similarly, Fu et al. [43] correctly identified malignant PNs from benign PNs with $89.8 \%$ sensitivity and $81.3 \%$ specificity. Broza et al. [34] evaluated over two dozen breath samples from 17 patients undergoing resection for suspicious PNs and was able to detect LC with $100 \%$ sensitivity and $80 \%$ 
specificity. Likewise, Shlomi et al. [74] was able to detect LC from a cohort of suspicious PNs with $75 \%$ sensitivity and $93 \%$ specificity.

One study examined the ability to predict the presence of suspicious pulmonary nodules on low-dose CT. Using a single unidentified VOC, Phillips et al. [62] predicted biopsy-proven lung cancer with $75.4 \%$ sensitivity and $85 \%$ specificity, and presence of suspicious pulmonary nodules with $80.1 \%$ sensitivity and $75.0 \%$ specificity.

\section{ii. Comorbidities}

Three studies aimed to characterize LC in the presence of comorbidities. In 2005, Poli et al. [67] found significantly higher levels of 2-methylpentane and isoprene and significantly lower levels of ethylbenzene and styrene in NSCLC patients vs COPD patients. Molina et al. [58] screened LC vs CM with $70 \%$ sensitivity and $61.1 \%$ specificity using 2,6-bis-1,1-dimethylethyl-4-(1-methyl-1-

phenylethyl)phenol. Wang et al. [80] examined breath samples from 484 subjects and was able to distinguish between LC and $\mathrm{CM}$ groups with a $0.701 \mathrm{AUC}$ using 1,2,4-trimethylbenzene. The authors concluded comorbidities may have significant interference in the selection of VOC markers for LC diagnosis.

Ten studies examined the ability to diagnose LC in the presence of comorbidities. In the presence of comorbidities, seven studies reported an overall decrease in diagnostic accuracy and two studies reported an overall increase. All studies reported a decrease in specificity in the presence of comorbidities. Five studies reported an increase in sensitivity in the presence of comorbidities. Dragonieri et al. [42] correctly recognized LC with an overall accuracy of $90 \%$ amongst $\mathrm{H}$ subjects, which decreased to $85 \%$ amongst $\mathrm{CM}$ subjects. D'Amico et al. [40] recorded $85 \%$ sensitivity and $100 \%$ specificity against $\mathrm{H}$ controls, which decreased overall to $92.8 \%$ sensitivity and $78.6 \%$ specificity against CM groups. Similarly, 3-way model of LC vs. H vs. CM recorded $89 \%$ sensitivity for LC and $75 \%$ specificity when compared with non-cancers. Shehada et al. [73] achieved $87 \%$ sensitivity and $82 \%$ specificity versus $\mathrm{H}$ groups, which increased overall to $92 \%$ sensitivity and $80 \%$ specificity versus CM groups. Molina et al. [58] correctly discriminated between LC and $\mathrm{H}$ subjects with $82.4 \%$ sensitivity and $88.7 \%$ specificity, which decreased overall to $86.8 \%$ sensitivity and $67.5 \%$ specificity against B subjects. Kort et al. [52] screened LC with $92.2 \%$ sensitivity and $51.2 \%$ specificity amongst $\mathrm{H}$ subjects, which decreased overall to $94.4 \%$ sensitivity and $32.9 \%$ specificity amongst B subjects. Gasparri et al. [46] considered whether the presence of comorbidities in LC patients could potentially affect VOC screening of $\mathrm{H}$ controls. With comorbidities, LC patients were diagnosed with $85 \%$ sensitivity and $88 \%$ specificity. Without comorbidities, LC patients were diagnosed with $76 \%$ sensitivity and $94 \%$ specificity. Two studies reported a decrease in sensitivity in the presence of comorbidities. Tirzite et al. [76] correctly diagnosed LC from $\mathrm{H}$ controls with $97.8 \%$ sensitivity and $68.8 \%$ specificity. Against B controls, sensitivity and specificity decreased to $88.9 \%$ and $66.7 \%$, respectively. Lu et al. [54] demonstrated a
94.2\% sensitivity and a $92.8 \%$ specificity for LC versus B controls. In a 3-way classification model between LC, $\mathrm{H}$, and $\mathrm{CM}$, performance decreased to $87.2 \%$ sensitivity and $80.6 \%$ specificity. One study reported no change in sensitivity in the presence of comorbidities. Wang et al. [81] demonstrated a $96.5 \%$ sensitivity and a $97.5 \%$ specificity between LC and B controls. In a 3-way classification model of LC vs. H vs. CM, sensitivity remained unchanged while specificity decreased to $87.5 \%$.

In summary, the presence of comorbidities on lung cancer diagnosis appears to increase sensitivity, decrease specificity, and decrease overall accuracy. Consequently, VOCs may hold greater potential for screening than diagnosis in patients with comorbidities.

\subsubsection{Classification}

Lung cancer is classified by histological and molecular subtype. The type of lung cancer can have significant implications for patient prognosis and therapeutic management [99]. The two major histological classifications are small cell lung cancer (SCLC) and non-small cell lung cancer (NSCLC). NSCLC accounts for roughly $85 \%$ of LC cases and mainly consists of adenocarcinoma (ADC), squamous cell carcinoma (SQC), and large cell carcinoma (LCC) [100]. NSCLC can be further subdivided into molecular subtypes based on the presence of genetic mutations, e.g. KRAS, TP53, Epidermal Growth Factor Receptor (EGFR), Anaplastic Lymphoma Kinase (ALK).

i. Histology

Two studies distinguished histological subtypes from controls. Oguma et al. [59] found that concentrations cyclohexane and xylene were significantly higher in patients with ADC or SCLC in controls, though the difference between controls and patients with SQC was less marked. Handa et al. [47] analyzed 115 VOC peaks from exhaled breath samples of 50 LC patients (including $32 \mathrm{ADC}$ ) and 39 healthy subjects. n-Dodecane was able to separate adenocarcinoma and healthy subjects with a sensitivity of $81.3 \%$ and a specificity of $89.7 \%$.

Three studies distinguished histological subtypes from each other. Song et al. [75] examined breath samples from 43 subjects with LC (including 19 ADC and 24 SQC) and 41 healthy controls via GC-MS. Patients with adenocarcinoma were found to have significantly elevated concentrations in 1-butanol and 3-hydroxy-2-butanone relative to SQC. Corradi et al. [39] studied breath samples from 71 patients with NSCLC (consisting of 48 ADC and 23 SQC) and 67 controls. Breath analysis revealed elevated concentrations of hexane in patients with ADC vs SQC. Fu et al. [43] examined breath samples from 97 cases and 88 healthy controls. Case participants consisted of 9 with SCLC and 88 NCLC, including 33 ADC and 32 SQC. Patients with SQC were found to have significant elevations in 4-hydroxyhexenal compared to adenocarcinoma. In addition, patients with SCLC were found to have significant elevations in the concentrations of 4 - hydroxynonenal, pentanone, and n-pentanal compared to patients with NSCLC.

ii. Mutations 
Two studies sought to distinguish molecular subtypes from controls. In a study by Handa et al. [47], LC patients with EGFR mutations had significantly higher levels of n-Dodecane compared to LC patients without EGFR mutations, with a sensitivity of $85.7 \%$ and a specificity of $78.6 \%$. In a study by Shlomi et al. [74], LC patients with EGFR mutations were discriminated from LC patients without EGFR mutations with a sensitivity of $79 \%$ and specificity of $85 \%$.

\subsubsection{Stage}

The extent of lung cancer is most commonly described using the international TNM-based staging system. Stages range from one to four (I through IV) and take into account the size of the primary tumor, the involvement of regional lymph nodes, and the presence or absence of distant metastatic spread. The lower the stage, the less the cancer has spread.

Three studies distinguished the stages of lung cancer from healthy controls. One approach distinguished between various stages and controls. Fu et al. [43] found the exhaled breath concentration of 2-butanone significantly higher in patients with stage I LC. A related study by Oguma et al. [59] found concentrations of cyclohexane and xylene increased significantly as the clinical stage of lung cancer advanced. In stage III patients, concentrations of xylene were significantly elevated. In stage IV patients, concentrations of cyclohexane and xylene were significantly elevated.

Three studies distinguished the stages of lung cancer from each other. $\mathrm{Fu}$ et al. [43] found the exhaled breath concentration of 2-butanone significantly lower in patients with stage I LC compared to patients with stages II-IV LC. Corradi et al. [39] detected significantly elevated concentrations of ethyl benzene in stages III+IV compared to stages I+II. Oguma et al. [59] found concentrations of cyclohexane and xylene increased significantly as the clinical stage of lung cancer advanced.

Two studies evaluated the ability to diagnose lung cancer by stages. A diagnostic model by Oguma et al. [59] was able to determine lung cancer with 75\% sensitivity and $78 \%$ specificity, which decreased slightly to $73 \%$ sensitivity and $78 \%$ specificity for early lung cancer. Lu et al. [54] analyzed 214 breath samples by e-nose and was able to correctly identify LC with $94.2 \%$ sensitivity and $92.8 \%$ specificity. The authors detected stage II with $97.9 \%$ sensitivity and $70 \%$ specificity, stage III with $82.8 \%$ sensitivity and $81.8 \%$ specificity, and stage IV with $83.2 \%$ sensitivity and $81.6 \%$ specificity.

Three studies evaluated the ability to diagnose early stage lung cancer versus late stage. Shehada et al. [73] was able to correctly distinguish early stage LC from late stage LC with $34.5 \%$ sensitivity and $95 \%$ specificity. Mazzone et al. [56] was able to correctly distinguish early stage LC from late stage LC with $81 \%$ sensitivity and $73 \%$ specificity. Peled et al. [60] achieved excellent distinction between early and advanced stages of NSCLC with $86 \%$ sensitivity and $88 \%$ specificity.

\subsection{Malignant Pleural Mesothelioma}

A total of 7 studies on MPM and VOCs were reviewed.
VOCs uniquely associated with MPM were identified in 3 studies. A total of 68 VOCs were reported as significant biomarkers for MPM-mainly consisting of aromatic compounds $(\sim 30.9 \%)$, alkanes $(\sim 22.1 \%)$, and alkenes $(\sim 11.8 \%)$ (Figure 3$)$. The most common VOCs were Toluene, Xylene, Acetophenone, 2-ethylhexanol, Hexane, Cyclohexane, Limonene, alpha-Pinene, and beta-Pinene, reported in at least 2 studies.

The ability of VOCs to determine malignancy was tested in 7 studies. Sensitivity and specificity ranged from $80-91 \%$ and $80-89.5 \%$, respectively. Diagnostic VOC models, ranging from 2 to 32 VOCs, were described and evaluated in 5 studies. Two studies characterized MPM with respect to healthy controls. de Gannero et al. [86] found cyclohexane alone to be highly discriminant for MPM against $\mathrm{H}$ controls.

\subsubsection{Asbestos Exposure}

Asbestos refers to a group of naturally occurring mineral fibers historically used in building materials and fabrics. Asbestos exposure (AEx) is a known cause of lung and pleural disease: inhaled asbestos fibers lead to oxidative stress and stimulate a protracted immune reaction that damages the pleura [101-103].

Two studies characterized MPM in the presence of asbestos exposure. In 2010, de Gennaro et al. [86] found cyclohexane to be highly discriminant for MPM against AEx controls. Lamote et al. [89] found cyclohexane and limonene highly discriminant for MPM against AEx controls.

Five studies assessed the ability to diagnose MPM in the presence of asbestos exposure. All three studies reported an increase in diagnostic accuracy and specificity. Two studies reported a decrease in sensitivity. A 3-way classification model of MPM vs. H vs. AEx by de Gennaro et al. [86] was able to discriminate for MPM with $84.6 \%$ sensitivity and $100 \%$ specificity. Giglio et al. [88] correctly predicted cancer with $100 \%$ specificity and $50 \%$ specificity on a prospective independent cohort of 5 AEx subjects. Lamote first distinguished MPM from $\mathrm{H}$ with $96 \%$ sensitivity and $67 \%$ specificity, which increased overall to $87 \%$ sensitivity and $86 \%$ specificity against the AEx group, and decreased overall to $87 \%$ sensitivity and $70 \%$ specificity against the pooled B group [90]. In a subsequent study, Lamote distinguished MPM from $\mathrm{H}$ controls with $89 \%$ sensitivity and $42 \%$ specificity, which increased to $87 \%$ sensitivity and $90 \%$ specificity against the AEx cohort [91]. One study reported an increase in sensitivity. Lamote et al. [89] obtained $67 \%$ sensitivity and $64 \%$ specificity against $\mathrm{H}$ subjects using electronic sensor array, which increased overall to $80 \%$ sensitivity and $64 \%$ specificity against AEx subjects. Using GC-MS, the authors obtained $64 \%$ sensitivity and $79 \%$ specificity against $\mathrm{H}$ subjects, which increased to $93 \%$ sensitivity and $100 \%$ specificity against AEx subjects.

In summary, diagnostic accuracy and specificity for MPM appears to increase given a history of asbestos exposure. These results suggest that VOCs screening may yield lower false positive rates in patients with a history of asbestos exposure. 


\subsubsection{Asbestos-related Disease}

Asbestos-related disease (ARD) can be classified as benign (asbestosis, benign pleural effusions, benign pleural plaques and diffuse pleural thickening) and malignant (lung cancer, mesothelioma of the pleura and/or peritoneum) [101-103].

Four studies tested the ability to diagnose MPM in the presence of asbestos-related disease, including fibrosis and/or asbestos plaques. Three studies report an increase in diagnostic accuracy in the presence of ARD, while two studies report a decrease in diagnostic accuracy. Three studies report an increase in specificity while one study reports a decrease in specificity. Three studies report no changes in sensitivity, while one study reports an increase in sensitivity. Chapman et al. [85] correctly classified MPM from H groups with $90 \%$ sensitivity and $91 \%$ specificity; in a three-way classification of MPM, ARD, and $\mathrm{H}$, the authors achieved $90 \%$ sensitivity and $88 \%$ specificity. Dragonieri et al. [87] correctly classified MPM with $85 \%$ accuracy against $H$ subjects, which decreased to $81 \%$ accuracy against ARD subjects and $80 \%$ accuracy in a 3-way classification model between MPM, ARD, and $\mathrm{H}$ groups. Lamote et al. [91] was able detect MPM from $\mathrm{H}$ controls with a $89 \%$ sensitivity and $42 \%$ specificity, which increased overall to $89 \%$ sensitivity and $73 \%$ specificity against the ARD cohort. Lamote et al. [89] obtained $67 \%$ sensitivity and $64 \%$ specificity against $\mathrm{H}$ subjects using electronic sensor array, which increased overall to $75 \%$ sensitivity and $64 \%$ specificity against ARD subjects. Using GC-MS, the authors obtained $64 \%$ sensitivity and $79 \%$ specificity against $\mathrm{H}$ subjects, which increased overall to $79 \%$ sensitivity and $80 \%$ specificity against ARD subjects. In a blinded validation cohort of 5 AEx individuals, a model by Gilio et al. [88] misclassified two patients found to have pleural plaques, suggesting a potential diagnostic challenge.

Two studies examined the ability to diagnose MPM in the presence of asbestos-related disease and asbestos exposure $(\mathrm{ARD}+\mathrm{AEx})$. Given the combination of ARD+AEx, one study reports a decrease in accuracy compared to AEx alone. Another study reports mixed results: a decrease in accuracy using GC-MS, and an increase in accuracy using e-nose. Both studies report an increase in diagnostic accuracy compared to ARD alone. Lamote et al. [91] was able to distinguish MPM from ARD with $89 \%$ sensitivity and $73 \%$ specificity, which increased overall to $94 \%$ sensitivity and $80 \%$ specificity against a pooled cohort of AEx+ARD subjects. In a follow up study, Lamote et al. [89] used GC-MS to discriminate MPM from ARD with $79 \%$ sensitivity and $80 \%$ specificity, which increased overall to $100 \%$ sensitivity and $91 \%$ specificity when both AEx and ARD groups were pooled. Using electronic sensor technology, the authors discriminated MPM patients from ARD with 75\% sensitivity and 64\% specificity. When AEx and ARD patients were pooled, diagnostic performance increased overall to $82 \%$ sensitivity and $55 \%$ specificity. The authors found diethyl ether and nonanal to be highly discriminant for MPM vs. AEx+ARD.

One study examined the determination of MPM in the setting of pulmonary disease. Lamote et al. [91] sampled breath from 330 participants with a history of asbestos exposure, asbestos-related disease, and/or pulmonary disease (e.g., COPD, cystic fibrosis, and lung cancer). The authors were detected MPM versus a pooled AEx + ARD cohort with 94\% sensitivity and $80 \%$ specificity, which decreased overall to $71.2 \%$ and $87 \%$ versus a $\mathrm{CM}$ cohort and $73 \%$ and $71 \%$ versus a LC cohort, respectively.

In summary, diagnostic accuracy and specificity for MPM appears to increase in the presence of asbestos-related disease and may be further increased by history of asbestos exposure.

\subsection{Esophageal Cancer}

A total of 6 studies on EC and VOCs were reviewed.

VOCs uniquely associated with EC were identified in 4 studies. A total of 35 VOCs were reported as significant biomarkers for EC - mainly consisting of aldehydes $(\sim 28.6 \%)$, aromatic compounds $(\sim 28.6 \%)$, and fatty acids $(\sim 20.0 \%)$ (Figure 3 ). Notably, alkanes and ketones were not detected by any study. The most common VOCs were Phenol, Hexanoic Acid, Methyl Phenol, Ethyl Phenol, Decanal, Butanal, Pentanoic Acid, and Butyric Acid, reported in at least 2 studies. Of note, cancer groups typically included both esophageal and gastric adenocarcinoma; these cancers are considered comparable subtypes that have frequently been grouped together in neoadjuvant chemotherapy trials. Markar et al. [95] analyzed concentrations of 5 highly predictive VOCs and found no significant differences between esophageal and gastric cancer patients.

The ability of VOCs to determine malignancy was tested in 6 studies. Sensitivity and specificity ranged from $80-91 \%$ and $80-89.5 \%$, respectively. Diagnostic VOC models, ranging from 4 to 8 discrete VOCs, were described and evaluated in 6 studies. Kumar et al. [93] noted exhaled breath concentrations of methanol, hexanoic acid, phenol, methyl phenol were clearly elevated in subjects with EC compared to H controls. A multicenter study by Markar et al. [95] was able diagnose esophagogastric cancer with $80 \%$ sensitivity and $81 \%$ specificity.

\subsubsection{Comorbidities}

One study sought to characterize EC in the presence of comorbidities. Kumar et al. [93] noted elevated breath concentrations of hexanoic acid, phenol, methyl phenol, and ethyl phenol in the EC group vs CM group.

Two studies tested the ability to diagnose EC in the presence of comorbidities. In the aforementioned study by Kumar et al., subsequent ROC analysis found the same 4 VOCs were able to discriminate EGC from CM with an AUC of 0.91. A subsequent study from the same team was able to discriminate between EC vs. $\mathrm{H}$ cohorts with $98 \%$ sensitivity and $91.7 \%$ specificity [94]. Between EC vs. B cohorts, sensitivity and specificity decreased to $87.5 \%$ and $82.9 \%$, respectively.

\subsubsection{Barrett's Esophagus}

Barrett's esophagus is a precursor to esophageal cancer and its early identification has been associated with improved survival [104]. 
Two studies examined the ability to diagnose Barrett's Esophagus. Chan et al. [92] 2016 analyzed 66 BE patients and $56 \mathrm{~B}$ with electronic sensor and achieved $82 \%$ sensitivity and $81 \%$ specificity. More recently, a prediction model by Peters et al. [96] correctly differentiated between EC and $\mathrm{H}$ controls with $57 \%$ sensitivity and $67 \%$ specificity.

One study examined the ability to diagnose Barrett's Esophagus in the presence of comorbidities. In the same Peters et al. [96] study mentioned above, sensitivity and specificity increased to $64 \%$ and $74 \%$ versus CM groups and $91 \%$ and $74 \%$ versus B groups, respectively.

\subsubsection{Stage}

The extent of esophageal cancer is described using an international TNM-based staging system similar to lung cancer.

One study evaluated the ability to diagnose esophageal cancer by stages. Zou et al. [97] analyzed breath samples from 29 EC cases and achieved a discriminant accuracy of $100 \%$, $71 \%, 86 \%$, and $93 \%$ for stage I, II, III, and IV, respectively. However, the study population is relatively small (There were $1,7,7$, and 14 patients in stage I, II, III, and IV respectively) so the value of discriminant accuracy at different tumor stages may be not quite accurate.

\section{Discussion}

Interest in the use of exhaled breath VOCs to determine thoracic malignancies is growing. Almost half of all studies included in our review were published within the past 5 years alone. In addition, electronic sensor accounts for the majority of lung cancer studies published within the same time period and are gaining popularity over GC-MS as the VOC detection method of choice. The reliable performance of electronic sensor array combined with its many practical strengths make it an appealing technology for point-of-care screening and diagnosis. Concurrently, recent studies are less likely to identify specific VOCs. This trend reflects a broader and more holistic shift in our approach to exhaled breath VOCs as complex, dynamic, and multidimensional biomarkers for malignant disease unlikely to be adequately characterized by discrete singular compounds. Still, identification of VOCs remains useful for elucidating metabolic pathways of disease, enriching our understanding of underlying cellular processes, and generating hypothesis for novel therapeutic interventions.

Exhaled breath VOCs in thoracic malignancies typically consisted of aromatics, aldehydes, alkanes, lipids, ketones, and sulfur-containing compounds. Aromatic compounds are usually considered to be pollutants from exogenous sources, including cigarette smoke, alcohol, and pollution. Most lung cancer patients have a long smoking history, and some studies found that certain aromatic compounds increased in the breath of smoking patients versus nonsmokers [50, 105]. These pollutants may result in peroxidative damage to PUFA, proteins, and DNA, leading to age-dependent diseases [106]. Aldehydes in the body come from 4 major sources [16]: (1) oxidation of fatty acids, where concentrations have been found to increase in concentration during inflammation and oxidative stress; (2) ethanol metabolism, where ethanol is degraded by alcohol dehydrogenase to produce acetaldehyde and subsequently oxidized by aldehyde dehydrogenase to acetate; (3) tobacco metabolism, where it's formation is catalyzed by cytochrome $\mathrm{P} 450$ as part of the detoxification process; and (4) cigarette smoke (e.g., formaldehyde, acetaldehyde, ethanal, propanal, butanal). In patients with esophageal cancer, genetic dysregulation of aldehyde metabolism has been observed [95]. Alkanes are largely produced by oxidation of fatty acids, particularly during the peroxidation of polyunsaturated fatty acids (e.g., ethane, pentane) [16]. Protein oxidation and fecal flora may also yield alkanes (e.g., propane, butane) [107]. Lipids are required for the membrane synthesis, potentially due to accelerated cell proliferation. The upregulation of fatty acids has been reported in esophageal cancer tissue [108, 109]. Ketones, like other hydrocarbons, are mainly generated via fatty acid oxidation. During times of fasting or starvation, hepatocytes produce acetone via decarboxylation of excess Acetyl-CoA secondary to lipid peroxidation by cytochrome $\mathrm{p} 450$. In the state of cachexia, typically under illness conditions such as cancer, protein metabolism would increase and result in higher levels of ketone bodies. In addition to endogenous ketones, some occur naturally in the environment and are absorbed by the body (e.g., 2-butanone) [16]. The presence of volatile sulfur compounds (e.g. dimethyl sulfide, dimethyl disulfide, methanethiol) is largely the result of incomplete metabolism of methionine [107].

\section{Conclusion}

There is considerable evidence to support the notion of exhaled breath VOCs to determine thoracic malignancies. A variety of breath sampling and analytical techniques have been able to determine the presence of lung cancer in particular. Similar studies on malignant pleural mesothelioma and esophageal cancer have begun to emerge within the past 10 years with comparable and equally encouraging results.

Despite their promise, the use of exhaled VOCs has not translated to routine clinical practice. Results between studies are widely inconsistent. Studies tend to manage covariates poorly and vary widely in methodology, making it difficult to generate consensus. Further, the lack of externally validated multicenter studies on independent cohorts remains a critical issue. In order to enhance reproducibility and facilitate the transition of exhaled VOCs into a clinical setting, the European Respiratory Society has published recommendations for standardization of sampling, analyzing and reporting of data [110]. Commercially available breath sampling devices offer several advantages over traditional sampling methods and may also further improve experimental validity [111]. The recently developed ReCIVA breath sampler is a quick and convenient device that is repeatable and provides the researcher with added control and functionality [112]. The use of standardized instrumentation to diagnose thoracic cancers via exhaled VOCs is an active area of 
research - two major ongoing studies funded by the United Kingdom National Health Service are of particular interest $[113,114]$. The Lung Cancer Indicator Detection (LuCID) study is an international multicenter prospective case-control cohort study which aims to identify an exhaled VOC biosignature that can accurately diagnose lung cancer. Exhaled breath will be sampled from up to 4000 patients with clinical suspicion of lung cancer. The PAN-cancer Early Detection (PAN) study is a prospective cross-sectional observational case-control study of up to 1500 participants evaluating whether breath VOCs can accurately distinguish between individuals with and without different cancer types, including esophagogastric cancer. The LuCID and PAN studies are the largest of their kind and may finally provide the insights necessary to move forward with a reliable and non-invasive biomarker for thoracic cancers.

\section{References}

[1] Creaney, J. and B. W. S. Robinson, Malignant Mesothelioma Biomarkers: From Discovery to Use in Clinical Practice for Diagnosis, Monitoring, Screening, and Treatment. Chest, 2017. 152 (1): p. 143-149.

[2] Kim, J. A. and P. M. Shah, Screening and prevention strategies and endoscopic management of early esophageal cancer. Chin Clin Oncol, 2017. 6 (5): p. 50.

[3] Hoffman, R. M. and R. Sanchez, Lung Cancer Screening. Med Clin North Am, 2017. 101 (4): p. 769-785.

[4] Rivera, M. P., A. C. Mehta, and M. M. Wahidi, Establishing the Diagnosis of Lung Cancer: Diagnosis and Management of Lung Cancer, 3rd ed: American College of Chest Physicians Evidence-Based Clinical Practice Guidelines. Chest, 2013. 143 (5, Supplement): p. e142S-e165S.

[5] Alsop, B. R. and P. Sharma, Esophageal Cancer. Gastroenterology Clinics of North America, 2016. 45 (3): p. 399-412.

[6] Shaheen, N. J., et al., ACG Clinical Guideline: Diagnosis and Management of Barrett's Esophagus. Am J Gastroenterol, 2016. 111 (1): p. 30-50; quiz 51.

[7] Arif, Q. and A. N. Husain, Malignant Mesothelioma Diagnosis. Arch Pathol Lab Med, 2015. 139 (8): p. 978-80.

[8] Kindler, H. L., et al., Treatment of Malignant Pleural Mesothelioma: American Society of Clinical Oncology Clinical Practice Guideline. J Clin Oncol, 2018. 36 (13): p. 1343-1373.

[9] Torre, L. A., R. L. Siegel, and A. Jemal, Lung Cancer Statistics. Adv Exp Med Biol, 2016. 893: p. 1-19.

[10] World Health Organization. Cancer Fact Sheet. 2018 [cited $20208 / 31]$.

[11] Brusselmans, L., et al., Breath analysis as a diagnostic and screening tool for malignant pleural mesothelioma: a systematic review. Translational lung cancer research, 2018. 7 (5): p. 520-536.

[12] Odgerel, C. O., et al., Estimation of the global burden of mesothelioma deaths from incomplete national mortality data.
Occup Environ Med, 2017.74 (12): p. 851-858.

[13] Pakzad, R., et al., The incidence and mortality of esophageal cancer and their relationship to development in Asia. Ann Transl Med, 2016.4 (2): p. 29.

[14] Jemal, A., et al., Global cancer statistics. CA Cancer J Clin, 2011. 61 (2): p. 69-90.

[15] American Cancer Society. Key Statistics for Esophageal Cancer. [cited 2020 8/31]; Available from: https://www.cancer.org/cancer/esophagus-cancer/about/key-st atistics.html.

[16] Hakim, M., et al., Volatile organic compounds of lung cancer and possible biochemical pathways. Chem Rev, 2012. 112 (11): p. 5949-66.

[17] Nardi-Agmon, I. and N. Peled, Exhaled breath analysis for the early detection of lung cancer: recent developments and future prospects. Lung Cancer (Auckl), 2017. 8: p. 31-38.

[18] Gordon, S. M., et al., Volatile organic compounds as breath biomarkers for active and passive smoking. 2002. 110 (7): p. 689-698.

[19] Pauling, L., et al., Quantitative analysis of urine vapor and breath by gas-liquid partition chromatography. Proc Natl Acad Sci U S A, 1971.68 (10): p. 2374-6.

[20] Arasaradnam, R. P., et al., Non-invasive exhaled volatile organic biomarker analysis to detect inflammatory bowel disease (IBD). 2016. 48 (2): p. 148-153.

[21] Dummer, J., et al., Analysis of biogenic volatile organic compounds in human health and disease. 2011. 30 (7): p. 960-967.

[22] Chambers, S. T., A. Scott-Thomas, and M. J. C. o. i. p.m. Epton, Developments in novel breath tests for bacterial and fungal pulmonary infection. 2012. 18 (3): p. 228-232.

[23] Phillips, M., et al., Volatile biomarkers of pulmonary tuberculosis in the breath. 2007. 87 (1): p. 44-52.

[24] Gahleitner, F., et al., Metabolomics pilot study to identify volatile organic compound markers of childhood asthma in exhaled breath. Bioanalysis, 2013. 5 (18): p. 2239-47.

[25] Montuschi, P., et al., The electronic nose in respiratory medicine. Respiration, 2013. 85 (1): p. 72-84.

[26] Fens, N., et al., Exhaled breath profiling enables discrimination of chronic obstructive pulmonary disease and asthma. Am J Respir Crit Care Med, 2009. 180 (11): p. 1076-82.

[27] Tisch, U., et al., Detection of Alzheimer's and Parkinson's disease from exhaled breath using nanomaterial-based sensors. 2013. 8 (1): p. 43-56.

[28] Peng, G., et al., Detection of lung, breast, colorectal, and prostate cancers from exhaled breath using a single array of nanosensors. Br J Cancer, 2010. 103 (4): p. 542-51.

[29] Phillips, M., et al., Prediction of breast cancer risk with volatile biomarkers in breath. Breast Cancer Res Treat, 2018. 170 (2): p. 343-350.

[30] Speiser, D., et al., Volatile organic compounds (VOCs) in exhaled breath of patients with breast cancer in a clinical setting. 2012. 83 (10). 
[31] Sun, X., et al., Detection of volatile organic compounds (VOCs) from exhaled breath as noninvasive methods for cancer diagnosis. 2016. 408 (11): p. 2759-2780.

[32] Bajtarevic A., et al., Noninvasive detection of lung cancer by analysis of exhaled breath. BMC Cancer. 2009 Sep 29; 9: 348.

[33] Buszewski B., et al., Identification of volatile lung cancer markers by gas chromatography-mass spectrometry: comparison with discrimination by canines. Anal Bioanal Chem. 2012 Jul; 404 (1): 141-6.

[34] Broza, Y. Y., et al., A nanomaterial-based breath test for short-term follow-up after lung tumor resection. Nanomedicine, 2013. 9 (1): p. 15-21.

[35] Cai X., et al., A Prediction Model with a Combination of Variables for Diagnosis of Lung Cancer. Med Sci Monit. 2017 Nov 25; 23: 5620-5629.

[36] Chang, Ji-Eun., et al., Analysis of volatile organic compounds in exhaled breath for lung cancer diagnosis using a sensor system." Sensors and Actuators B: Chemical 255 (2018): 800-807.

[37] Chen X., et al., A study of the volatile organic compounds exhaled by lung cancer cells in vitro for breath diagnosis. Cancer. 2007 Aug 15; 110 (4): 835-44.

[38] Chen X., et al., A Non-invasive detection of lung cancer combined virtual gas sensors array with imaging recognition technique. Conf Proc IEEE Eng Med Biol Soc. 2005; 2005: 5873-6.

[39] Corradi, M., et al., Exhaled breath analysis in suspected cases of non-small-cell lung cancer: a cross-sectional study. J Breath Res, 2015.9 (2): p. 027101.

[40] D'Amico, A., et al., An investigation on electronic nose diagnosis of lung cancer. Lung Cancer, 2010. 68 (2): p. 170-6.

[41] Di Natale C., et al., Lung cancer identification by the analysis of breath by means of an array of non-selective gas sensors. Biosens Bioelectron. 2003 Sep; 18 (10): 1209-18.

[42] Dragonieri, S., et al., An electronic nose in the discrimination of patients with non-small cell lung cancer and COPD. Lung Cancer, 2009. 64 (2): p. 166-70.

[43] Fu, X. A., et al., Noninvasive detection of lung cancer using exhaled breath. Cancer Med, 2014.3 (1): p. 174-81.

[44] Fuchs P., et al., Breath gas aldehydes as biomarkers of lung cancer. Int J Cancer. 2010 Jun 1; 126 (11): 2663-70.

[45] Gaspar EM., et al., Organic metabolites in exhaled human breath--a multivariate approach for identification of biomarkers in lung disorders. J Chromatogr A. 2009 Apr 3; 1216 (14): 2749-56.

[46] Gasparri, R., et al., Volatile signature for the early diagnosis of lung cancer. J Breath Res, 2016. 10 (1): p. 016007.

[47] Handa, H., et al., Exhaled breath analysis for lung cancer detection using ion mobility spectrometry. PLoS One, 2014. 9 (12): p. e114555.

[48] Huang CH, et al., A Study of Diagnostic Accuracy Using a Chemical Sensor Array and a Machine Learning Technique to Detect Lung Cancer. Sensors (Basel). 2018 Aug 28; 18 (9): 2845 .
[49] Hubers AJ., et al., Combined sputum hypermethylation and eNose analysis for lung cancer diagnosis. J Clin Pathol. 2014 Aug; 67 (8): 707-11.

[50] Kischkel, S., et al., Breath biomarkers for lung cancer detection and assessment of smoking related effects - confounding variables, influence of normalization and statistical algorithms. 2010. 411 (21-22): p. 1637-1644.

[51] Kononov A., et al. Online breath analysis using metal oxide semiconductor sensors (electronic nose) for diagnosis of lung cancer. J Breath Res. 2019 Oct 23; 14 (1): 016004.

[52] Kort, S., et al., Multi-centre prospective study on diagnosing subtypes of lung cancer by exhaled-breath analysis. Lung Cancer, 2018. 125: p. 223-229.

[53] Ligor M., et al., Determination of volatile organic compounds in exhaled breath of patients with lung cancer using solid phase microextraction and gas chromatography mass spectrometry. Clin Chem Lab Med. 2009; 47 (5): 550-60.

[54] Lu, B., et al., A Novel Framework with High Diagnostic Sensitivity for Lung Cancer Detection by Electronic Nose. Sensors (Basel), 2019. 19 (23).

[55] Machado RF., et al., Detection of lung cancer by sensor array analyses of exhaled breath. Am J Respir Crit Care Med. 2005 Jun 1; 171 (11): 1286-91.

[56] Mazzone, P. J., et al., Exhaled breath analysis with a colorimetric sensor array for the identification and characterization of lung cancer. J Thorac Oncol, 2012. 7 (1): p. 137-42.

[57] Mazzone, P. J., et al., Diagnosis of lung cancer by the analysis of exhaled breath with a colorimetric sensor array. Thorax. 2007 Jul; 62 (7): 565-8.

[58] Molina, A., et al., Identification of metabolomics panels for potential lung cancer screening by analysis of exhaled breath condensate. J Breath Res, 2016. 10 (2): p. 026002.

[59] Oguma, T., et al., Clinical contributions of exhaled volatile organic compounds in the diagnosis of lung cancer. PLoS One, 2017. 12 (4): p. e0174802.

[60] Peled, N., et al., Non-invasive breath analysis of pulmonary nodules. J Thorac Oncol, 2012. 7 (10): p. 1528-33.

[61] Phillips M., et al., Prediction of lung cancer using volatile biomarkers in breath. Cancer Biomark. 2007; 3 (2): 95-109.

[62] Phillips, M., T. L. Bauer, and H. I. Pass, A volatile biomarker in breath predicts lung cancer and pulmonary nodules. J Breath Res, 2019. 13 (3): p. 036013.

[63] Phillips M., et al., Blinded Validation of Breath Biomarkers of Lung Cancer, a Potential Ancillary to Chest CT Screening. PLoS One. 2015 Dec 23; 10 (12): e0142484.

[64] Phillips M., et al., Detection of lung cancer using weighted digital analysis of breath biomarkers. Clin Chim Acta. $2008 \mathrm{Jul}$ 17; 393 (2): 76-84.

[65] Phillips M., et al., Detection of lung cancer with volatile markers in the breath. Chest. 2003 Jun; 123 (6): 2115-23.

[66] Phillips M., et al., Volatile organic compounds in breath as markers of lung cancer: a cross-sectional study. Lancet. 1999 Jun 5; 353 (9168): 1930-3. 
[67] Poli, D., et al., Exhaled volatile organic compounds in patients with non-small cell lung cancer: cross sectional and nested short-term follow-up study. Respir Res, 2005. 6: p. 71.

[68] Rocco R., et al., BIONOTE e-nose technology may reduce false positives in lung cancer screening programmes $\dagger$. Eur J Cardiothorac Surg. 2016 Apr; 49 (4): 1112-7; discussion 1117.

[69] Rudnicka J., et al., Searching for selected VOCs in human breath samples as potential markers of lung cancer. Lung Cancer. 2019 Sep; 135: 123-129.

[70] Rudnicka J., et al., Determination of volatile organic compounds as biomarkers of lung cancer by SPME-GC-TOF/MS and chemometrics. J Chromatogr B Analyt Technol Biomed Life Sci. 2011 Nov 15; 879 (30): 3360-6.

[71] Sakumura Y., et al., Diagnosis by Volatile Organic Compounds in Exhaled Breath from Lung Cancer Patients Using Support Vector Machine Algorithm. Sensors (Basel). 2017 Feb 4; 17 (2): 287.

[72] Schallschmidt $\mathrm{K}$, et al., Comparison of volatile organic compounds from lung cancer patients and healthy controls-challenges and limitations of an observational study. J Breath Res. 2016 Oct 12; 10 (4): 046007.

[73] Shehada, N., et al., Silicon Nanowire Sensors Enable Diagnosis of Patients via Exhaled Breath. ACS Nano, 2016. 10 (7): p. 7047-57.

[74] Shlomi, D., et al., Detection of Lung Cancer and EGFR Mutation by Electronic Nose System. J Thorac Oncol, 2017. 12 (10): p. 1544-1551.

[75] Song, G., et al., Quantitative breath analysis of volatile organic compounds of lung cancer patients. Lung Cancer, 2010. 67 (2): p. 227-31.

[76] Tirzite, M., et al., Detection of lung cancer with electronic nose and logistic regression analysis. J Breath Res, 2018. 13 (1): p. 016006 .

[77] Tirzite M., et al., Detection of lung cancer in exhaled breath with an electronic nose using support vector machine analysis. J Breath Res. 2017 Aug 21; 11 (3): 036009.

[78] Ulanowska, A., et al., The application of statistical methods using VOCs to identify patients with lung cancer. J Breath Res, 2011.5 (4): p. 046008.

[79] van de Goor R., et al., Training and Validating a Portable Electronic Nose for Lung Cancer Screening. J Thorac Oncol. 2018 May; 13 (5): 676-681.

[80] Wang, M., et al., Confounding effect of benign pulmonary diseases in selecting volatile organic compounds as markers of lung cancer. J Breath Res, 2018. 12 (4): p. 046013.

[81] Wang, Y., et al., The analysis of volatile organic compounds biomarkers for lung cancer in exhaled breath, tissues and cell lines. Cancer Biomark, 2012. 11 (4): p. 129-37.

[82] Westhoff M., et al., Ion mobility spectrometry for the detection of volatile organic compounds in exhaled breath of patients with lung cancer: results of a pilot study. Thorax. 2009 Sep; 64 (9): 744-8.

[83] Yu H., et al., Solid phase microextraction for analysis of alkanes and aromatic hydrocarbons in human breath. J Chromatogr B Analyt Technol Biomed Life Sci. 2005 Nov 5; 826 (1-2): 69-74.
[84] Zou Y., et al., Optimization of volatile markers of lung cancer to exclude interferences of non-malignant disease. Cancer Biomark. 2014; 14 (5): 371-9.

[85] Chapman, E. A., et al., A breath test for malignant mesothelioma using an electronic nose. 2012. 40 (2): p. 448-454.

[86] de Gennaro, G., et al., Chemical characterization of exhaled breath to differentiate between patients with malignant plueral mesothelioma from subjects with similar professional asbestos exposure. 2010. 398 (7-8): p. 3043-3050.

[87] Dragonieri, S., et al., An electronic nose distinguishes exhaled breath of patients with Malignant Pleural Mesothelioma from controls. Lung Cancer, 2012.75 (3): p. 326-31.

[88] Gilio, A., et al., Breath Analysis for Early Detection of Malignant Pleural Mesothelioma: Volatile Organic Compounds (VOCs) Determination and Possible Biochemical Pathways. Cancers, 2020. 12 (5): p. 1262.

[89] Lamote, K., et al., Breath analysis by gas chromatography-mass spectrometry and electronic nose to screen for pleural mesothelioma: a cross-sectional case-control study. 2017.8 (53): p. 91593.

[90] Lamote, K., et al., Detection of malignant pleural mesothelioma in exhaled breath by multicapillary column/ion mobility spectrometry (MCC/IMS). J Breath Res, 2016. 10 (4): p. 046001.

[91] Lamote, K., et al., Exhaled breath to screen for malignant pleural mesothelioma: a validation study. Eur Respir J, 2017. $50(6)$.

[92] Chan, D. K., et al., Breath Testing for Barrett's Esophagus Using Exhaled Volatile Organic Compound Profiling With an Electronic Nose Device. Gastroenterology, 2017. 152 (1): p. 24-26.

[93] Kumar, S., et al., Selected ion flow tube mass spectrometry analysis of exhaled breath for volatile organic compound profiling of esophago-gastric cancer. Anal Chem, 2013. 85 (12): p. 6121-8.

[94] Kumar, S., et al., Mass Spectrometric Analysis of Exhaled Breath for the Identification of Volatile Organic Compound Biomarkers in Esophageal and Gastric Adenocarcinoma. Ann Surg, 2015. 262 (6): p. 981-90.

[95] Markar, S. R., et al., Assessment of a Noninvasive Exhaled Breath Test for the Diagnosis of Oesophagogastric Cancer. JAMA Oncol, 2018.4 (7): p. 970-976.

[96] Peters, Y., et al., Detection of Barrett's oesophagus through exhaled breath using an electronic nose device. 2020. 69 (7): p. 1169-1172.

[97] Zou, X., et al., Exhaled gases online measurements for esophageal cancer patients and healthy people by proton transfer reaction mass spectrometry. J Gastroenterol Hepatol, 2016. 31 (11): p. 1837-1843.

[98] National Lung Screening Trial Research Team., et al., Reduced lung-cancer mortality with low-dose computed tomographic screening. 2011. 365 (5): p. 395-409.

[99] Lemjabbar-Alaoui, H., et al., Lung cancer: Biology and treatment options. Biochimica et biophysica acta, 2015. 1856 (2): p. 189-210. 
[100] Noone, A., et al., SEER cancer statistics review, 1975-2015. 2018. 4 .

[101] Stanton, MF., et al., Mechanisms of mesothelioma induction with asbestos and fibrous glass. 1972. 48 (3): p. 797-821.

[102] Cugell, D. W. and D. W. J. C. Kamp, Asbestos and the pleura: a review. 2004. 125 (3): p. 1103-1117.

[103] Wagner, J. C., et al., Diffuse pleural mesothelioma and asbestos exposure in the North Western Cape Province. 1960. 17 (4): p. 260-271.

[104] Bhat, S. K., et al., Oesophageal adenocarcinoma and prior diagnosis of Barrett's oesophagus: a population-based study. Gut, 2015. 64 (1): p. 20-5.

[105] Horvath, I., et al., Exhaled biomarkers in lung cancer. 2009. 34 (1): p. 261-275.

[106] Haick, H., et al., Assessment, origin, and implementation of breath volatile cancer markers. 2014. 43 (5): p. 1423-1449.

[107] Miekisch, W., J. K. Schubert, and G. F. J. C. c. a. Noeldge-Schomburg, Diagnostic potential of breath analysis-focus on volatile organic compounds. 2004. 347 (1-2): p. 25-39.
[108] Wang, L., et al., 1H-NMR based metabonomic profiling of human esophageal cancer tissue. Molecular Cancer, 2013. 12 (1): p. 25 .

[109] Tan, B., et al., Metabonomics identifies serum metabolite markers of colorectal cancer. Journal of proteome research, 2013. 12 (6): p. 3000-3009.

[110] Horváth, I., et al., A European Respiratory Society technical standard: exhaled biomarkers in lung disease. Eur Respir J, 2017. 49 (4).

[111] Beale, D. J., et al., A review of analytical techniques and their application in disease diagnosis in breathomics and salivaomics research. 2017. 18 (1): p. 24.

[112] ReCIVA Breath Sampler. [cited 2020 September 21]; Available from: https://www.owlstonemedical.com/products/reciva/.

[113] Van Der Schee, M., et al., MS29. 04 LuCID Exhaled Breath Analysis. 2018. 13 (10): p. S302.

[114] PAN cancer study. [cited 2020 September 21]; Available from: https://www.owlstonemedical.com/clinical-pipeline/pan/. 